\title{
TAME FRÉCHET STRUCTURES FOR AFFINE KAC-MOODY GROUPS*
}

\author{
WALTER FREYN ${ }^{\dagger}$
}

\begin{abstract}
We construct holomorphic loop groups and their associated affine Kac-Moody groups and prove that they are tame Fréchet manifolds; furthermore we study the adjoint action of these groups. These results form the functional analytic core for a theory of affine Kac-Moody symmetric spaces, that will be developed in forthcoming papers. Our construction also solves the problem of complexification of completed Kac-Moody groups: we obtain a description of complex completed Kac-Moody groups and, using this description, deduce constructions of their non-compact real forms.
\end{abstract}

Key words. Loop group, loop algebra, affine Kac-Moody group, affine Kac-Moody algebra, tame Fréchet space, completion.

AMS subject classifications. 22E67, 20G44.

1. Introduction. In this article we define and investigate a class of tame Fréchet Lie groups and tame Fréchet Lie algebras, whose members are constructed as certain functional analytic completions of affine Kac-Moody groups and affine Kac-Moody algebras respectively. Affine Kac-Moody algebras were introduced and first studied in the 60's independently by V. G. Kac [Kac68], R. V. Moody [Moo69] and I. L. Kantor [Kan70]; they were shown to be natural infinite dimensional generalizations of finite dimensional simple Lie algebras, sharing important aspects of their structure and representation theory [Car02, Kac90, MP95]. Originally, affine Kac-Moody algebras are defined abstractly using a presentation, consisting of a set of generators subject to certain relations, called the Chevalley-Serre relations, which are derived from some affine Cartan matrix; this presentation generalizes verbatim similar presentations for finite dimensional simple Lie algebras. Associated to affine Kac-Moody algebras are affine Kac-Moody groups; starting from some affine Cartan matrix, they can be defined either in a functorial way or via a presentation, which is, roughly speaking, a group theoretical version of the one, used to define Kac-Moody algebras [KP85, Tit87, Tit89]. We call the resulting affine Kac-Moody algebras and affine Kac-Moody groups algebraic in contrast to their functional analytic completions, which we call analytic affine Kac-Moody algebras and affine Kac-Moody groups respectively. Affine Kac-Moody algebras and their associated (affine) Kac-Moody groups are intensively studied and by now well understood; especially, a complete classification of complex affine KacMoody algebras and their real forms is known. To a large part the importance of affine Kac-Moody algebras comes from an alternative second description: they can be constructed as 2-dimensional extensions of (possibly twisted) loop algebras. The existence of this second explicit description distinguishes affine Kac-Moody algebras in the much wider class of Kac-Moody algebras; these loop algebras can be viewed as spaces of periodic functions from $\mathbb{R}$ into some finite dimensional simple Lie algebra, satisfying suitable regularity conditions. More precisely, following E. Heintze and C. Gross [HG12], for some simple Lie algebra $\mathfrak{g}$ and some automorphism of finite order $\sigma$ of $\mathfrak{g}$, we define the (twisted) loop algebras

$L(\mathfrak{g}, \sigma)=\{f: \mathbb{R} \longrightarrow \mathfrak{g} \mid f(t+2 \pi)=\sigma f(t)$ and $f$ satisfies some regularity conditions $\}$.

\footnotetext{
* Received November 22, 2012; accepted for publication August 20, 2013.

$\dagger$ Fachbereich Mathematik, Technische Universität Darmstadt, Schloßgartenstr. 7, 64289 Darmstadt, Germany (freyn@mathematik.tu-darmstadt.de).
} 
Typically $\mathfrak{g}$ is a simple Lie algebra and $\sigma$ a diagram automorphism (possibly the identity), hence of order 1,2 or 3 . Nevertheless remark that in the construction of affine Kac-Moody symmetric spaces more general choices for $\mathfrak{g}$ and $\sigma$ appear [Fre13c]. In this language, the algebraic affine Kac-Moody algebras consist of loops whose Fourier series expansion is finite, when viewed as periodic map from $\mathbb{R}$ into a finite dimensional simple Lie algebra $\mathfrak{g}$. Similarly, algebraic Kac-Moody groups consist of loops into some finite dimensional Lie group, whose Fourier series is finite for some fixed choice of representation (see for example [PS86], chapter III).

Various completions of affine Kac-Moody algebras and affine Kac-Moody groups appear naturally in applications to problems in mathematical physics, geometry and analysis. Examples include solutions of general relativity [BM87], integrable systems [SW85], moduli spaces of CMC-surfaces [Kob11], isometry groups of symmetric spaces [Fre13a], isometry groups of isoparametric submanifolds [Ter89] or isometry groups of (twin) buildings or twin cities [Fre12b].

In these and similar applications, a recurring challenge is, that the original algebraic affine Kac-Moody algebras and affine Kac-Moody groups have the disadvantage of not admitting a manifold structure, excluding the use of strong functional analytic tools. Hence, to achieve a manifold structure one considers completions of the set of algebraic loops, whose details most often are specified by the requirements of the application. Typical completions are $C^{k}$-differentiable loops, $H^{k}$-Sobolev-loops, smooth loops or sometimes real analytic loops.

In the loop algebra formulation, real forms may be defined explicitly by imposing special reality condition on the loops. In general one distinguishes two classes of real forms, called almost-compact real forms or almost-split real forms (for details and classifications see [Rou89a, Rou89b, HG12]). For example, the compact real form can be viewed as a 2-dimensional extension of loops into the compact real form $\mathfrak{g}_{c}$ of $\mathfrak{g}$.

These families of different completions are very satisfactory for the the compact real form or more generally for almost compact real forms, as the important 2-dimensional extension which gives the Kac-Moody group then can be defined far a wide variety of regularity conditions [PS86]; however, the construction of this extension fails for most completions in the complex case and in consequence for all (almost split) real forms. This leads to huge technical difficulties in applications, where these forms are needed.

We solve this basic problem by the introduction of some intermediate completion, consisting of (possibly twisted) holomorphic loops on $\mathbb{C}^{*}$ into complex finite dimensional simple Lie groups $G$. If $G$ is semisimple, the resulting complete affine Kac-Moody groups contain the algebraic affine Kac-Moody groups as dense subgroups and are themselves contained as dense subgroups in the well-known completions of $C^{1}-$ or $H^{k}$-loops. In this setting, the 2-dimensional extensions can be defined for all forms, the complex forms, the almost compact ones and the almost split ones.

We show, that our completion of affine Kac-Moody groups, using holomorphic functions, is the largest setting, admitting these central extensions. We will use this setting in forthcoming work to construct affine Kac-Moody symmetric spaces; this work answers affirmatively a conjecture, which was originally formulated by C.-L. Terng in her seminal paper [Ter95] and then broadened by E. Heintze into a wide research program [Hei08] and lays thus the foundations for affine Kac-Moody geometry [Fre12a]. Affine Kac-Moody symmetric spaces are the closest infinite dimensional analogue to finite dimensional Riemannian symmetric spaces. As in the finite dimensional case, there are affine Kac-Moody symmetric spaces of the compact type and of 
the non-compact type. These two types correspond to various forms of finite dimensional (semi-)simple Lie groups and their quotients respectively. Especially, symmetric spaces of the non-compact type are diffeomorphic to vector spaces. Similar results hold for affine Kac-Moody symmetric spaces.

We prove, that these new groups admit in the real as in the complex case useful manifold structures as tame Fréchet manifolds in the sense of Hamilton [Ham82]. Let us recall, that Fréchet spaces are close generalizations of Banach spaces. They are defined as locally convex, Hausdorff, topological vector spaces, whose topology is generated by a countable family of seminorms, in contrast to Banach spaces, where only one single seminorm is used. In comparison to Banach spaces, handling Fréchet spaces poses additional challenges. First, from a purely technical point of view, the uncountably sequences of norms have to be controlled, then dual spaces of Fréchet spaces are in general not Fréchet and most importantly, there is no useful inverse function theorem for maps between Fréchet spaces. To resolve this last issue, one restricts attention to subclasses of Fréchet spaces, that admit inverse function theorems. The most common such class is the one of tame Fréchet spaces, introduced in [Ham82]. Tame Fréchet spaces are defined using careful estimates of the sequences of seminorms. We start in section 2 to review the theory of tame Fréchet structures following the presentation given by Richard Hamilton [Ham82]; the main purpose of this section is, to collect and restate some basic results, which we will need at several places in a formulation, which is slightly different from the one, found in the literature. We explain, that tame Fréchet space are, essentially, spaces of Banach-space valued holomorphic functions.

Then, in section 3 , we study spaces of holomorphic maps on $\mathbb{C}^{*}$ with various target spaces and prove, that they are tame Fréchet spaces; using the loop algebra representation of affine Kac-Moody algebras, these spaces reappear as the basic building blocks in our construction of the tame Fréchet structures on affine Kac-Moody algebras and affine Kac-Moody groups. Hence, these estimates form a crucial ingredient to our theory. In section 4 we then prove, that holomorphic loop algebras are tame Fréchet Lie algebras; recalling, that a tame Fréchet Lie algebra is a tame Fréchet vector space $V$ such that the endomorphisms $a d(v): V \rightarrow V$ are tame linear maps for all $v \in V$, tame Fréchet Lie algebras appear as the natural generalization of Banach Lie algebras to tame Fréchet spaces. Hence the key challenge is, to find appropriate estimates for the norms of the Lie brackets $\|[v, w]\|_{n}$ in terms of the norms $\|v\|_{n+k}$ and $\|w\|_{n+k}$ for all $n \in \mathbb{N}$ and some fixed integer $k \geq 0$ similar to the estimates, used in the definition of Banach algebras. Furthermore we prove some central results about the tameness of Kac-Moody groups, which may be summarized as follows:

THEOREM 1.1. Groups of holomorphic loops and their real forms are tame Fréchet Lie groups.

The usually used approach to establish the existence of manifold structures on loop groups is, to check first that the exponential function is a local diffeomorphism, leading to the definition of a chart in some open set of the identity and then using (left) translation, to get charts on the whole loop group. This approach, described in [PS86], is for example used for $C^{k}$-differentiable loops, $H^{k}$-Sobolev-loops or smooth loops. Nevertheless, this approach fails for holomorphic loop groups, essentially due to Liouvilles-theorem, stating, that bounded holomorphic functions on $\mathbb{C}$ are constant; thus there are no holomorphic functions on $\mathbb{C}^{*}$, whose image is contained in bounded sets of the identity $U \subset \mathfrak{g}$ of the (finite dimensional simple) Lie algebra and $V \subset G$ of the finite dimensional simple Lie group, such that $\exp : U \rightarrow V$ is a diffeomorphism. 
Moreover, we show that for the loop groups of semisimple Lie groups the exponential map is not a local diffeomorphism; hence there is also no other way, to define charts in the Lie algebra via the exponential map.

To achieve the desired result, that groups of holomorphic loops are tame Fréchet Lie groups, we need to establish various technical results about Fréchet manifolds and tame Fréchet submanifolds. Our proof describes the holomorphic loop groups as subgroups of certain vector spaces of holomorphic maps, which we prove to carry a structure as a tame Fréchet space. Using the main result of [Fre12c], we establish first, that our loop groups are tame Fréchet submanifolds of co-finite type and then, that they are tame Fréchet manifolds.

In section 5 we extend the theory of polar actions from the classical Hilbert space setting [Ter95] to our setting of tame Fréchet spaces; recall, that the action of group $G$ on a vector space $V$ is called polar if there is a subspace $\Sigma \in V$, called a section, intersecting each $G$-orbit $G * x, x \in V$ orthogonally. Polar actions of finite dimensional Lie groups are classified and it is shown, that they correspond up to orbit equivalence to the isotropy representations of finite dimensional Riemannian symmetric spaces [BCO03]. A closely related, but infinite dimensional theory of proper Fredholm polar actions on Hilbert spaces was then introduced in [HPTT95] and further studied in [Ter95, HL99, Gro00], where it was established, that these proper Fredholm polar actions on Hilbert spaces are closely related to the adjoint action of certain Hilbert-space completions of affine Kac-Moody algebras. To extend the result to the setting of tame Fréchet vector spaces, the key point is, to establish that each orbit really intersects the section. As in the finite dimensional case these actions are related to the isotropy representations of affine Kac-Moody symmetric spaces; nevertheless, the relation is a little more complicated, due to the more complicated structure of the isotropy representations of affine Kac-Moody symmetric spaces; details will be given in forthcoming work.

In the final section 7 we turn our attention to the description of the affine KacMoody groups, that are constructed as two-dimensional extensions of our groups of holomorphic loops and prove that they are tame Fréchet Lie groups. Here we use a result of B. Popescu [Pop05], stating that certain fiber bundles over tame Fréchet manifolds are tame Fréchet spaces again. Essentially, we prove, that all complex forms, real forms and quotient spaces of affine Kac-Moody groups, that we will need in the construction of affine Kac-Moody symmetric spaces in [Fre13a] are tame Fréchet manifolds. Furthermore we prove, that the quotient spaces, we will identify with affine Kac-Moody symmetric spaces of the non-compact type in [Fre13a] are (as in the finite dimensional case) diffeomorphic to vector spaces (theorem 7.6 and theorem 7.7).

\section{Tame Fréchet manifolds.}

2.1. Fréchet spaces. In this introductory section we collect some standard results about Fréchet spaces, Fréchet manifolds and Fréchet Lie groups. Further details and omitted proofs can be found in Hamilton's article [Ham82], section I.1 .

Definition 2.1 (Fréchet space). A Fréchet vector space is a locally convex topological vector space which is complete, Hausdorff and metrizable.

LEMma 2.2 (Metrizable topology). A topology on a vector space is metrizable iff it can be defined by a countable collection of seminorms.

Proof. For the proof see [Ham82]. 
Let us give some examples:

ExAmPLE 2.3 (Fréchet spaces).

1. Every Banach space is a Fréchet space. The 'countable collection' of norms contains just one element.

2. Let $\operatorname{Hol}(\mathbb{C}, \mathbb{C})$ denote the space of holomorphic functions $f: \mathbb{C} \longrightarrow \mathbb{C}$. Let furthermore $K_{n}$ be a sequence of simply connected compact sets in $\mathbb{C}$, such that $K_{n} \subset K_{n+1}$ and $\bigcup K_{n}=\mathbb{C}$. Let $\|f\|_{n}:=\sup _{z \in K_{n}}|f(z)|$. Then $\operatorname{Hol}\left(\mathbb{C}, \mathbb{C} ;\|\|_{n}\right)$ is a Fréchet space.

3. More generally, for every Riemann surface $S$ the sheaf of holomorphic functions carries a Fréchet structure which is defined similarly as in the special case $S=\mathbb{C}$ discussed in the second example.

Definition 2.4. A Fréchet manifold is a (possibly infinite dimensional) manifold with charts in a Fréchet space such that the chart transition functions are smooth.

While it is possible to define Fréchet manifolds in this way, there are two strong impediments to the development of analysis and geometry of those spaces:

1. In general there is no inverse function theorem for smooth maps between Fréchet spaces. For counterexamples and examples showing features special to Fréchet spaces, see [Ham82].

2. The dual space of a Fréchet space is not a Fréchet space as soon as the Fréchet space is not Banach.

The solution to the first problem is based on picking some subclass of Fréchet spaces, called tame Fréchet spaces, that allow for an inverse function theorem. The key in its construction is a more refined control of the sequences of norms. Using this control, the famous Nash-Moser inverse function theorem is established. In the next sections we formalize those concepts.

Let us note that there are other ways to deal with these analytic problems. A recently proposed method is the concept of bounded Fréchet geometry developed by Olaf Müller [Mül06, Mül08].

The solution to the second problem consists in avoiding dual spaces. Let us remark that the theory could also be formulated by defining dual spaces as direct limit locally convex topological vector spaces.

2.2. Tame Fréchet spaces. The central problem for all further structure theory of Fréchet spaces is a better control of the collection of seminorms. For a single Fréchet space this is done by the concept of a grading; much deeper is the question of comparing collections of seminorms on two different Fréchet spaces: for two Fréchet spaces $F$ and $G$ and a map $\varphi: F \longrightarrow G$ this is done by imposing estimates similar in spirit to the concept of quasi isometries relating the sequences of norms $\|\varphi(f)\|_{n}$ and $\|f\|_{m}$, leading to the concept of "tame" maps. The property of a Fréchet space to be tame is defined then by "tame equivalence" to some model space $\Sigma(B)$, which is essentially a space of holomorphic functions; the standard reference for these considerations is the article [Ham82]. We are slightly sharpening the concept of "tame equivalence" introduced by Hamilton to define $(r, b, C(n))$-equivalence and emphasize the nature of tame spaces as a generalization of spaces of holomorphic functions.

The prerequisite for estimating norms under maps between Fréchet spaces is a comparison of the norms on the Fréchet space itself: 
Definition 2.5 (grading). Let $F$ be a Fréchet space. A grading on $F$ is a collection of seminorms $\left\{\|\|_{n}, n \in \mathbb{N}_{0}\right\}$ that define the topology and satisfy

$$
\|f\|_{0} \leq\|f\|_{1} \leq\|f\|_{2} \leq\|f\|_{3} \leq \ldots
$$

Lemma 2.6 (Constructions of graded Fréchet spaces).

1. A closed subspace of a graded Fréchet space is a graded Fréchet space.

2. Direct sums of graded Fréchet spaces are graded Fréchet spaces.

Proof. For the proof see [Ham82], section II.1.

Every Fréchet space admits a grading. Let $\left(F,\|\|_{n, n \in \mathbb{N}}\right)$ be a Fréchet space. Then

$$
\left(\widetilde{F},\|\|_{n, n \in \mathbb{N}}\right)
$$

such that $\widetilde{F}=F$ as a set and

$$
\widetilde{\|}\left\|_{n}:=\sum_{i=1}^{n}\right\| \|_{i}
$$

is a graded Fréchet space. The Fréchet topologies of $F$ and $\widetilde{F}$ coincide. Obviously other gradings my be defined on $F$, for example by introducing positive coefficients in equation 1. The existence of a grading is thus not a property of the Fréchet space as a metrizable topological space, but an additional structure.

Definition 2.7 (Tame equivalence of gradings). Let $F$ be a graded Fréchet space, $r, b \in \mathbb{N}$ be positive integers and $C(n) \in \mathbb{R}^{+}, n \in \mathbb{N}$ a sequence of positive real numbers. The two gradings $\left\{\|\|_{n}\right\}$ and $\{\widetilde{\|\|}\}$ are called $(r, b, C(n))$-equivalent iff

$$
\|f\|_{n} \leq C(n) \widetilde{\|f\|_{n+r}} \text { and } \widetilde{\|f\|_{n}} \leq C(n)\|f\|_{n+r} \text { for all } n \geq b .
$$

They are called tame equivalent iff they are $(r, b, C(n))$-equivalent for some $(r, b, C(n))$.

The following example is basic; tame Fréchet spaces will be defined as Fréchet spaces, that are sufficiently close to the spaces of this example.

ExAmple 2.8. Let $B$ be a Banach space with norm \|\|$_{B}$. Denote by $\Sigma(B)$ the space of all exponentially decreasing sequences $\left\{f_{k}\right\}, k \in \mathbb{N}_{0}$ of elements of $B$. On this space, we can define different gradings:

$$
\begin{aligned}
\|f\|_{l_{1}^{n}} & :=\sum_{k=0}^{\infty} e^{n k}\left\|f_{k}\right\|_{B}, \\
\|f\|_{l_{\infty}^{n}} & :=\sup _{k \in \mathbb{N}_{0}} e^{n k}\left\|f_{k}\right\|_{B} .
\end{aligned}
$$

Lemma 2.9. On the space $\Sigma(B)$ the two gradings $\|f\|_{l_{1}^{n}}$ and $\|f\|_{l_{\infty}^{n}}$ are tame equivalent.

Proof. For the proof see [Ham82].

Let $F, G, G_{1}$ and $G_{2}$ denote graded Fréchet spaces. 
Definition 2.10 (Tame linear map). Let $r, b \in \mathbb{N}$ be positive integers and $C(n) \in \mathbb{R}^{+}, n \in \mathbb{N}$ a sequence of positive real numbers. A linear map $\varphi: F \longrightarrow G$ is called $(r, b, C(n))$-tame if it satisfies the inequality

$$
\|\varphi(f)\|_{n} \leq C(n)\|f\|_{n+r} \quad \text { for all } f \in F .
$$

The map $\varphi: F \longrightarrow G$ is called tame iff it is $(r, b, C(n))$-tame for some $(r, b, C(n))$.

Tameness of maps depends on the grading. To see this, let us look at the following variant of an example of [Ham82]:

EXAMPLE 2.11. Let $\mathcal{P}$ be the space of entire holomorphic functions periodic with period $2 \pi i$ and bounded in each left half-plane $P_{n}:=\{z \in \mathbb{C} \mid \Re(z) \leq n\}$. Define $L: \mathcal{P} \longrightarrow \mathcal{P}$ by $L f(z)=f(2 z)$.

1. Define first the grading on $\mathcal{P}$ by:

$$
\|f\|_{n}=\sup \{|f(z)|: \Re(z)=n\} .
$$

Then $\|L f\|_{n} \leq\|f\|_{2 n}$, hence $L$ is not tame.

2. Define now the grading on $\mathcal{P}$ by

$$
\|f\|_{n}=\sup \left\{|f(z)|: \Re(z)=2^{n}\right\} .
$$

Then $\|L f\|_{n} \leq\|f\|_{n+1}$, hence $L$ is $(1,0,1)$-tame.

Clearly the two gradings are not tame equivalent.

Definition 2.12 (Tame isomorphism). A map $\varphi: F \longrightarrow G$ is called a tame isomorphism iff it is a linear isomorphism and $\varphi$ and $\varphi^{-1}$ are tame maps.

Definition 2.13 (Tame direct summand). $F$ is a tame direct summand of $G$ iff there exist tame linear maps $\varphi: F \longrightarrow G$ and $\psi: G \longrightarrow F$ such that $\psi \circ \varphi: F \longrightarrow F$ is the identity.

Definition 2.14 (Tame Fréchet space). $F$ is a tame Fréchet space iff there is a Banach space $B$ such that $F$ is a tame direct summand of $\Sigma(B)$.

LEMma 2.15 (Constructions of tame Fréchet spaces).

1. A tame direct summand of a tame Fréchet space is tame.

2. A Cartesian product of two tame Fréchet spaces is tame.

Proof. Compare lemmata 1.3.3 and 1.3.4 of [Ham82].

EXAMPLE 2.16. This example is used in section 3.

- The space $\Sigma\left(\mathbb{C}^{2}\right.$, Eucl $)$ of exponentially decreasing sequences of elements in $\mathbb{C}^{2}$ where $\mathbb{C}^{2}$ is equipped with the Euclidean norm is a tame Fréchet space.

- The space $\Sigma\left(\mathbb{C}^{2}\right.$, Sup $)$ of exponentially decreasing sequences of elements in $\mathbb{C}^{2}$ where $\mathbb{C}^{2}$ is equipped with the supremum-norm $\left\|\left(c_{1}, c_{1}^{\prime}\right)\right\|_{B}:=\sup \left(\left|c_{1}\right|,\left|c_{1}^{\prime}\right|\right)$ is a tame Fréchet space.

Let us remind, that tame Fréchet spaces are essentially spaces of holomorphic functions (see also [Fre12c]).

Lemma 2.17. Let $B$ be a complex Banach space. The space $\Sigma(B)$ of exponentially decreasing sequences in $B$ is isomorphic to the space $\operatorname{Hol}(\mathbb{C}, B)$ of $B$-valued holomorphic functions. 
Proof. Let first $f \in \operatorname{Hol}(\mathbb{C}, B)$ be a holomorphic function and let

$$
f(z)=\sum_{k=0}^{\infty} f_{k} z^{k}
$$

be its Taylor series expansion. The coefficients $f_{k}$ are elements of $B$. We have to show that the sequence $\left(f_{k}\right), k \in \mathbb{N}$ is an exponentially decreasing sequence. To deduce the necessary estimates, we use the $l_{\infty}$-norm and the following three ingredients:

1. As $f(z)$ is an entire function, the expansion $\|f(z)\|=\sum_{n=0}^{\infty} f_{k} z^{k}$ converges for all $z \in \mathbb{C}$ - hence $\sup _{z \in B\left(0, e^{n}\right)}|f(z)|<\infty$ of all $n$.

2. Differentiation of $f$ yields for the coefficient $f_{k}$ the identity: $f_{k}=\frac{1}{k !} f^{(k)}(0)$.

3. The Cauchy inequality [BG91], 2.1.20: Let $f$ be holomorphic on $B(0, r)$. Then

$$
\left|f^{(k)}(0)\right| \leq k ! \frac{\sup _{z \in B(0, r)}|f(z)|}{r^{k}}
$$

Combining these ingredients we get for any $n \in \mathbb{N}$

$$
\sup _{k}\left|f_{k}\right| e^{n k}=\sup \left|\frac{f^{(k)}(0)}{k !}\right| e^{k n} \leq \sup _{k} \frac{e^{k n}}{k !}\left|k ! \frac{\sup _{z \in B\left(0, e^{n}\right)}|f(z)|}{e^{n k}}\right|=\sup _{z \in B\left(0, e^{n}\right)}|f(z)| \leq \infty .
$$

Hence we find, that $\|f\|_{l_{\infty}^{n}}<\infty$ and the sequence $\left(f_{k}\right), k \in \mathbb{N}$ is exponentially decreasing.

Conversely let $\left(f_{k}\right)_{k \in \mathbb{N}}, f_{k} \in B$ be an exponentially decreasing sequence. We have a prove, that the power series

$$
f(z):=\sum_{k=0}^{\infty} f_{k} z^{k}
$$

defines an entire $B$-valued holomorphic function. To do this, we show, that $f(z)$ is bounded on any compact ball $B\left(0, e^{n}\right)$. Let $z \in B\left(0, e^{n}\right)$ then

$$
|f(z)|=\left|\sum_{k} f_{k} z^{k}\right| \leq \sum_{k}\left|f_{k}\right|\left|z^{k}\right| \leq \sum\left|f_{k}\right| e^{k n}<\infty .
$$

Thus $f(z)$ defines a holomorphic function on $B\left(0, e^{n}\right)$. As this is true for all $n$, the result follows.

Example 2.18. Let $B:=\mathbb{C}$. Then lemma 2.17 means just

$$
\Sigma(\mathbb{C}):=\left\{\left(a_{k}\right)_{k \in \mathbb{N}_{0}} \in \mathbb{C}\left|\sum_{k}\right| a_{k} \mid e^{k n}<\infty \forall n \in \mathbb{N}\right\} .
$$

and $\Sigma(\mathbb{C})=\operatorname{Hol}(\mathbb{C}, \mathbb{C})$.

There are many different examples of tame Fréchet spaces (compare example 2.3).

ExAmPle 2.19 (tame Fréchet spaces).

1. Every Banach space is a tame Fréchet space. The 'countable collection' of norms contains just one element. 
2. Let $\operatorname{Hol}(\mathbb{C}, \mathbb{C})$ denote the space of holomorphic functions $f: \mathbb{C} \longrightarrow \mathbb{C}$. Let furthermore $K_{n}:=B\left(0, e^{n}\right) \subset \mathbb{C}$ be the sequence of open balls around 0 of radius $n \in \mathbb{N}$. Let $\|f\|_{n}:=\sup _{z \in K_{n}}|f(z)|$. Then $\operatorname{Hol}\left(\mathbb{C}, \mathbb{C} ;\|\|_{n}\right)$ is a Fréchet space.

For proofs and additional examples see [Ham82], part II. In section 3 we study in detail the tame Fréchet spaces of holomorphic functions which we need for the construction of Kac-Moody symmetric spaces.

Let us now introduce the notion of a tame Fréchet Lie algebra:

Definition 2.20 (Tame Fréchet Lie algebra). A Fréchet Lie algebra $\mathfrak{g}$ is a tame Lie algebra iff it is a tame vector space and $\operatorname{ad}(g)$ is a tame linear map for every $g \in \mathfrak{g}$.

The condition on $\operatorname{ad}(X)$ assures the tame structure to be invariant under the adjoint action. It may be replaced by the equivalent condition

$$
\|[f, g]\|_{n} \leq C(n)\|f\|_{n+k}\|g\|_{n+k}
$$

for all $n>b, k \in \mathbb{N}$ and some sequence of real numbers $C(n), n \in \mathbb{N}$. We clearly have the following class of examples:

EXAmple 2.21. Any Banach Lie algebra (for a definition see [Chu12], p.79) is a tame Fréchet Lie algebra. Indeed, if $F$ is a Banach space with norm $\|\cdot\|$, then equation 2 simplifies to

$$
\|[f, g]\| \leq C\|f\|\|g\|
$$

Corollary 2.22. Any finite dimensional Lie algebra is a tame Fréchet Lie algebra.

EXAmPLE 2.23. The realizations of the loop algebras $L(\mathfrak{g}, \sigma)$ are tame Fréchet Lie algebras for $H^{0}$-Sobolev loops, smooth loops and holomorphic loops - compare section 6 .

Up to now all maps, we studied were linear maps. Let us now proceed by a short review of some nonlinear tame Fréchet objects:

Definition 2.24. A nonlinear map $\Phi: U \subset F \longrightarrow G$ is called $(r, b, C(n))$-tame iff it satisfies the inequality

$$
\|\Phi(f)\|_{n} \leq C(n)\left(1+\|f\|_{n+r}\right) \forall n>b .
$$

$\Phi$ is called tame iff it is $(r, b, C(n))$-tame for some $(r, b, C(n))$.

ExAmple 2.25. Suppose $F$ and $G$ are Banach space (hence the collection of norms consists of one norm) and $\Phi: F \longrightarrow G$ is a $\left(r_{1}, b_{1}, C_{1}\right)$-tame isomorphism with a $\left(r_{2}, b_{2}, C_{2}\right)$-tame inverse $\Phi^{-1}$. If $b_{1} \geq 2$ and $b_{2} \geq 2$ the condition on the norms vanishes. If $b_{1}=r_{1}=b_{2}=r_{2}=0$ we get

$$
\|\Phi(f)\| \leq C_{1}(1+\|f\|) \quad \text { and } \quad\left\|\Phi^{-1}(g)\right\| \leq C_{2}(1+\|g\|) .
$$

After some manipulations we find:

$$
\frac{1}{C_{2}}\|f\|-1 \leq\|\Phi(f)\| \leq C_{1}(1+\|f\|)
$$


which is similar to the definition of a quasi isometry [BBI01]

Lemma 2.26 (Construction of tame maps).

1. Let $\Phi: U \subset F \longrightarrow G_{1} \times G_{2}$ be a tame map. Let $\pi_{i}, i=1,2$ be the projections $\pi_{i}: G_{1} \times G_{2} \longrightarrow G_{i}, i=1,2$. The maps

$$
\Phi_{i}:=\pi_{i} \circ \Phi: U \longrightarrow G_{i}
$$

are tame as well.

2. Let $\Phi_{i}: U \subset F \longrightarrow G_{i}, i \in\{1,2\}$ be $\left(r_{i}, b_{i}, C_{i}(n)\right)$-tame maps. Then the map

$$
\Phi:=\left(\Phi_{1}, \Phi_{2}\right): U \longrightarrow G_{1} \times G_{2}
$$

is $\left(\max \left(r_{1}, r_{2}\right), \max \left(b_{1}, b_{2}\right), C_{1}(n)+C_{2}(n)\right)$-tame.

Proof.

1. Projections onto a direct factor are $\left(0,0,(1)_{n \in \mathbb{N}}\right)$-tame. The composition of tame maps is tame. Thus $\Phi_{i}$ is tame.

2. Let $f \in U \subset F$.

$$
\begin{aligned}
\|\Phi(f)\|_{n} & =\|\Phi(f)\|_{n}^{1}+\|\Phi(f)\|_{n}^{2} \leq \\
& \leq C_{1}(n)\left(1+\|f\|_{n+r_{1}}\right)+C_{2}(n)\left(1+\|f\|_{n+r_{2}}\right) \leq \\
& \leq C_{1}(n)\left(1+\|f\|_{n+\max \left(r_{1}, r_{2}\right)}\right)+C_{2}(n)\left(1+\|f\|_{n+\max \left(r_{1}, r_{2}\right)}\right)= \\
& \leq\left(C_{1}(n)+C_{2}(n)\right)\left(1+\|f\|_{n+\max \left(r_{1}, r_{2}\right)}\right)
\end{aligned}
$$

for all $n \geq \max \left(b_{1}, b_{2}\right)$.

\subsection{Tame Fréchet manifolds.}

Definition 2.27 (Tame Fréchet manifold). A tame Fréchet manifold is a Fréchet manifold with charts in a tame Fréchet space such that the chart transition functions are smooth tame maps.

EXAMPLE 2.28. Every Banach manifold is a tame Fréchet manifold.

Definition 2.29. Let $M$ and $N$ be two tame Fréchet manifolds modeled on $F$ resp. $G$. A map $f: M \longrightarrow N$ is tame iff for every pair of charts $\psi_{i}: V_{i} \subset N \longrightarrow V_{i}^{\prime}$ and $\varphi_{j}: U_{i} \subset M \longrightarrow U_{i}^{\prime}$, the map $\psi_{i} \circ f \circ \varphi_{j}^{-1}$ is tame whenever it is defined.

For the construction of tame structures on Kac-Moody groups we need the following notion, introduced in [Fre13a]:

Definition 2.30 (Tame Fréchet submanifold of co-finite type). Let $n \in \mathbb{N}$. A subset $M \subset F$ is a $n$-codimensional smooth submanifold of $F$ iff for every $m \in M$ there are open sets $U(m) \subset F, V(m) \subset G \times \mathbb{R}^{n}$ and a tame Fréchet chart $\varphi_{m}$ : $U(m) \longrightarrow V(m) \subset G \times \mathbb{R}^{n}$ such that

$$
\varphi_{M}(M \cap U(m))=G \cap V(m) .
$$

Lemma 2.31. A tame submanifold of co-finite type is a tame Fréchet manifold.

This result was proved in [Fre12c]. 
Lemma 2.32. Let $M \subset F$ be a tame Fréchet submanifold of co-finite type. Let $H$ be a tame Fréchet space. A map $\varphi_{M}: H \longrightarrow M$ is tame if it is tame as a map $\varphi_{F}: H \rightarrow F$, where $\varphi_{F}$ is defined via the embedding $M \subset F$.

This means, that we chose the tame structure on a tame Fréchet submanifold of co-finite type compatible with the tame structure on the vector the manifold is embedded in. We need this result in the proof that affine Kac-Moody groups carry a tame structure.

Proof. Let $\varphi$ be tame as a map $\varphi_{F}: H \rightarrow F$. Then the concatenation $\varphi_{i} \circ \varphi$ is tame for any chart $\varphi$ of $M$. Thus $\varphi$ is tame as a map into $M$.

The technical reason for working in the category of tame Fréchet spaces and tame maps is the following Nash-Moser inverse function theorem. We cite the version due to R. Hamilton (see [Ham82]).

TheOREM 2.33 (Nash-Moser inverse function theorem). Let $F$ and $G$ be tame Fréchet spaces and $\Phi: U \subseteq F \longrightarrow G$ a smooth tame map. Suppose that the equation for the derivative $D \Phi(f) h=k$ has a unique solution $h=V \Phi(f) k$ for all $f \in U$ and all $k$ and that the family of inverses $V \Phi: U \times G \longrightarrow F$ is a smooth tame map. Then $\Phi$ is locally invertible, and each local inverse $\Phi^{-1}$ is a smooth tame map.

A description of this theorem a proof and some of its applications is the subject of the article [Ham82]. In comparison to the classical Banach inverse function theorem the important additional assumption is that the invertibility of the differential is assumed not only in a single point $p$ but in a small neighborhood $U$ around $p$. This additional condition is necessary [Ham82] because in contrast to the Banach space situation it is not true in the Fréchet space case that the existence of an invertible differential in one point leads to invertibility in a neighborhood. Let us note the following result [Ham82], theorem 3.1.1. characterizing the family of smooth tame inverses.

Theorem 2.34. Let $L:(U \subseteq F) \times H \longrightarrow K$ be a smooth tame family of linear maps. Suppose that the equation

$$
L(f) h=k
$$

has a unique solution $h$ for all $f$ and $k$ and that the family of inverses $V(f) k=h$ is continuous and tame as a map from $K$ toH Then $V$ is also a smooth tame map

$$
V:(U \subseteq F) \times K \longrightarrow H .
$$

We discuss these construction in more detail in [Fre13b].

3. Tame Fréchet spaces for Kac-Moody symmetric spaces. In this section we prove the tameness of certain Fréchet spaces of holomorphic functions on $\mathbb{C}^{*}$. We show first, that the space $\operatorname{Hol}\left(\mathbb{C}^{*}, \mathbb{C}\right)$ is a tame Fréchet space; then we extend this result to the space $\operatorname{Hol}\left(\mathbb{C}^{*}, V_{\mathbb{C}}\right)$, where $V_{\mathbb{C}}$ denotes a complex vector space. We use these results in section 4.2 for the construction of tame Fréchet affine Kac-Moody algebras and their Kac-Moody groups via the loop algebra realization (resp. loop group realization).

Recall, that $\Sigma(\mathbb{C})=\operatorname{Hol}(\mathbb{C}, \mathbb{C})$ is a tame Fréchet space. As a consequence also the space $F:=\operatorname{Hol}\left(\mathbb{C}, \mathbb{C}^{n}\right)$ is a tame Fréchet space.

The proof of R. Hamilton makes strong use of the following observation: 
Lemma 3.1. Let $F:=\operatorname{Hol}(\mathbb{C}, \mathbb{C})$. The two gradings

$$
\|f\|_{L_{1}^{n}}:=\frac{1}{2 \pi} \int_{\partial B_{n}}|f(z)| d z
$$

and

$$
\|f\|_{L_{\infty}^{n}}:=\sup _{z \in B_{n}}\|f(z)\|
$$

are tame equivalent.

Proof. [Ham82].

Our aim is now to prove that $\operatorname{Hol}\left(\mathbb{C}^{*}, \mathbb{C}\right)$ is a tame Fréchet space. Let us introduce some notation:

\section{Notation 3.1 .}

- Let $A_{n}$ denote the annulus $A_{n}:=\left\{z \in \mathbb{C}^{*}\left|e^{-n} \leq\right| z \mid \leq e^{n}\right\}$ and define the boundaries of $A_{n}$ by

$$
\partial A_{n}^{+}:=\left\{z|| z \mid=e^{n}\right\} \quad \text { and } \quad \partial A_{n}^{-}:=\left\{z|| z \mid=e^{-n}\right\},
$$

- Let $A_{n}^{\prime}$ denote the set $A_{n}^{\prime}:=\{z \in \mathbb{C} \mid-n \leq \Re(z) \leq n, 0 \leq \Im(z) \leq 2 \pi i\}$,

- Let $B_{n}$ denote the closed disc $B_{n}:=\left\{z \in \mathbb{C}|| z \mid \leq e^{n}\right\}$.

At some points we apply the Cauchy integral formula and the maximum principle for holomorphic functions.

We start with our version of lemma 3.1. The proof of our result is considerably more involved because holomorphic functions on $\mathbb{C}^{*}$ may be unbounded in the limit $|z| \rightarrow \infty$ and $|z| \rightarrow 0$.

Lemma 3.2. Let $F:=\operatorname{Hol}\left(\mathbb{C}^{*}, \mathbb{C}\right)$. The two gradings

$$
\|f\|_{L_{\infty}^{n}}:=\sup _{z \in A_{n}}|f(z)|
$$

and

$$
\|f\|_{L_{1}^{n}}:=\frac{1}{2 \pi} \sup \left\{\int_{\partial A_{n}^{+}}|f(z)| d z, \int_{\partial A_{n}^{-}}|f(z)| d z\right\}
$$

are tame equivalent.

Proof.

1. We show: $\|f\|_{L_{1}^{n}} \leq\|f\|_{L_{\infty}^{n}}$.

$$
\begin{aligned}
\|f\|_{L_{1}^{n}} & =\frac{1}{2 \pi} \sup \left\{\int_{\partial A_{n}^{+}}|f(z)| d z, \int_{\partial A_{n}^{-}}|f(z)| d z\right\} \leq \\
& \leq \frac{1}{2 \pi} \sup \left\{\int_{\partial A_{n}^{+}} \sup _{\zeta \in A_{n}^{+}}|f(\zeta)| d z, \int_{\partial A_{n}^{-}} \sup _{\zeta \in A_{n}^{-}}|f(\zeta)| d z\right\} \leq \\
& \leq \sup \left\{\sup _{z \in \partial A_{n}^{+}}|f(z)|, \sup _{z \in \partial A_{n}^{-}}|f(z)|\right\} \leq \\
& \leq \sup _{z \in A_{n}}|f(z)|=\|f\|_{L_{\infty}^{n} .}
\end{aligned}
$$


2. We show: $\|f\|_{L_{\infty}^{n}} \leq \frac{2}{r}\|f\|_{L_{1}^{n}}$.

To this end, we identify the space $\operatorname{Hol}\left(\mathbb{C}^{*}, \mathbb{C}\right)$ with the space $\operatorname{Hol}_{2 \pi i}(\mathbb{C}, \mathbb{C})$ of $2 \pi i$ periodic functions. Under this identification $A_{n}$ is identified with $A_{n}^{\prime}$.

$$
\begin{aligned}
\|f\|_{L_{\infty}^{n}} & =\sup _{z \in A_{n}^{\prime}}|f(z)|= \\
& =\sup _{z \in A_{n}^{\prime}}\left|\frac{1}{2 \pi i}\left\{\int_{n+r}^{n+r+2 \pi i} \frac{f(\zeta)}{z-\zeta} d \zeta-\int_{-n-r}^{-n-r+2 \pi i} \frac{f(\zeta)}{z-\zeta} d \zeta\right\}\right| \leq \\
& =\sup _{z \in A_{n}^{\prime}}\left|\frac{1}{2 \pi i}\left\{\int_{n+r}^{n+r+2 \pi i} \frac{f(\zeta)}{z-\zeta} d \zeta|+| \int_{-n-r}^{-n-r+2 \pi i} \frac{f(\zeta)}{z-\zeta} d \zeta\right\}\right| \leq \\
& \leq \sup _{z \in A_{n}^{\prime}} \frac{1}{2 \pi}\left\{\int_{n+r}^{n+r+2 \pi i}\left|\frac{f(\zeta)}{r}\right| d \zeta+\int_{-n-r}^{-n-r+2 \pi i}\left|\frac{f(\zeta)}{r}\right| d \zeta\right\}= \\
& =\sup _{z \in A_{n}^{\prime}} \frac{1}{2 \pi r}\left\{\int_{n+r}^{n+r+2 \pi i}|f(\zeta)| d \zeta+\int_{-n-r}^{-n-r+2 \pi i}|f(\zeta)| d \zeta\right\} \leq \\
& \leq \frac{2}{r} \frac{1}{2 \pi} \sup \left\{\int_{n+r}^{n+r+2 \pi i}|f(\zeta)| d \zeta, \int_{-n-r}^{-n-r+2 \pi i}|f(\zeta)| d \zeta\right\}= \\
& =\frac{2}{r}\|f\|_{L_{\infty}^{n+r}}
\end{aligned}
$$

We now prove, that $\operatorname{Hol}\left(\mathbb{C}^{*}, \mathbb{C}\right)$ is a tame Fréchet space.

Lemma 3.3. $F:=\operatorname{Hol}\left(\mathbb{C}^{*}, \mathbb{C}\right)$ is a tame Fréchet space.

Recall from definition 2.14, that we have to find a Banach space $B$, such that $\operatorname{Hol}\left(\mathbb{C}^{*}, \mathbb{C}\right)$ is a tame direct summand of $\Sigma(B)$. We choose $B=\mathbb{C}^{2}$. This

Proof. Expand $f$ into its Laurent series expansion $f:=\sum_{k \in \mathbb{Z}} c_{k} z^{k}$ and set $f_{0}^{+}:=\sum_{k \in \mathbb{N}_{0}} c_{k} z^{k}$ and $f^{-}:=\sum_{-k \in \mathbb{N}} c_{k} z^{k}$. Clearly $f_{0}^{+}(z)$ and $f^{-}\left(\frac{1}{z}\right)$ are holomorphic functions on $\mathbb{C}$. Let

$$
\begin{aligned}
\varphi: \operatorname{Hol}\left(\mathbb{C}^{*}, \mathbb{C}\right) & \longrightarrow \Sigma\left(\mathbb{C}^{2}\right) \\
f & \mapsto\left(\left\{c_{k}\right\}_{k \geq 0},\left\{c_{k}\right\}_{k<0}\right) .
\end{aligned}
$$

We use the notation $\widetilde{c}_{k}:=\left(c_{k}, c_{-k}\right) \subset \mathbb{C}^{2}$ and equip $\mathbb{C}^{2}$ with the supremum-norm.

1. We show: $\|f\|_{L_{\infty}^{n}} \leq\left\|\left\{\widetilde{c}_{k}\right\}\right\|$.

$$
\begin{aligned}
\|f\|_{L_{\infty}^{n}} & =\sup _{z \in A_{n}}|f(z)| \leq \\
& \leq \sup _{z \in A_{n}}\left\{\left|f_{0}^{+}(z)\right|+\left|f^{-}(z)\right|\right\} \leq \\
& \leq 2 \sup _{z \in A_{n}}\left\{\sup \left\{\left|f_{0}^{+}(z)\right|,\left|f^{-}(z)\right|\right\}\right\}= \\
& =2 \sup \left\{\sup _{z \in A_{n}}\left|f_{0}^{+}(z)\right|, \sup _{z \in A_{n}}\left|f^{-}\left(\frac{1}{z}\right)\right|\right\}= \\
& =2 \sup \left\{\left\|f_{0}^{+}\right\|_{L_{\infty}^{n}},\left\|f^{-}\right\|_{L_{\infty}^{n}}\right\} \leq \\
& \leq 2 \sup \left\{\left\|\left\{c_{k}\right\}\right\|_{L_{1}^{n}}, \|\left\{c_{-k} \|_{L_{1}^{n}}\right\}\right\} \leq \\
& \leq 2\left\|\left\{\widetilde{c}_{k}\right\}\right\|_{L_{\infty}^{n}}
\end{aligned}
$$


2. We show: $\left\|\left\{c_{k}\right\}\right\|_{L_{\infty}^{n}} \leq\|f\|_{L_{1}^{n}}$.

$$
\begin{aligned}
\left\|\left\{\widetilde{c}_{k}\right\}\right\|_{L_{\infty}^{n}} & =\sup _{k} e^{n k}\left|\widetilde{c}_{k}\right|= \\
& =\sup _{k} e^{n k}\left|\sup \left\{c_{k}, c_{-k}\right\}\right|= \\
& \leq \sup \left\{\sup _{k} e^{n k}\left|c_{k}\right|, \sup _{k} e^{n k}\left|c_{-k}\right|\right\} \leq \\
& \leq \sup \left\{\sup _{k} e^{n k} \frac{1}{2 \pi}\left|\int_{n}^{n+2 \pi i} e^{-k z} f(z) d z\right|, \sup _{k} e^{n k} \frac{1}{2 \pi}\left|\int_{-n}^{-n+2 \pi i} e^{k z} f(z)\right|\right\} \leq \\
& \leq \sup \left\{\sup _{k} \frac{1}{2} \int_{n}^{n+2 \pi i}\left|e^{n k} e^{-k n}\right|\left|e^{-k i \Im(z)}\right||f(z)| d z,\right. \\
& \leq \sup _{k}\left\{\sup _{k} \frac{1}{2} \int_{n}^{n+2 \pi i}|f(z)| d z, \sup _{k} \frac{1}{2 \pi} \int_{-n}^{-n+2 \pi i}|f(z)| d z\right\} \leq \\
& \left.\leq \sup _{-n}\left\{\sup _{k} \frac{1}{2} \int_{n}^{n+2 \pi i} \sup _{\zeta \in \partial A_{n}^{\prime}+}|f(\zeta)| d z, \sup _{k} \frac{1}{2 \pi} \int_{-n}^{-n+2 \pi i} e^{-k n}|| e^{k i \Im(z)}|| f(z) \mid d z\right\} \leq \sup _{n}|f(\zeta)| d z\right\}= \\
& =\sup _{\zeta \in A_{n}^{+}}\left\{\sup _{\zeta \in \partial A_{n}^{\prime}}|f(z)|, \sup _{\zeta \in \partial A_{n}^{\prime}-}|f(z)|\right\} \leq \\
& \leq \sup _{\zeta \in A_{n}^{\prime}|f(\zeta)|}=\|f\|_{L_{\infty}^{n}} \quad
\end{aligned}
$$

We use the following result in the proof that an affine Kac-Moody algebra is a tame Fréchet Lie algebra (cf. definition 2.20):

Lemma 3.4. The differential

$$
\frac{d}{d z}: \operatorname{Hol}\left(\mathbb{C}^{*}, \mathbb{C}\right) \longrightarrow \operatorname{Hol}\left(\mathbb{C}^{*}, \mathbb{C}\right), \quad f \mapsto f^{\prime}
$$

is a tame linear map.

For the proof of lemma 3.4 we need the following technical result:

Lemma 3.5. Let $f$ be analytic on a closed disc $\bar{D}\left(z_{0}, R\right), R>0$. Let $0<R_{1}<R$. Denote by $\|f\|_{R}$ the supremum norm of $f$ on the circle of radius $R$. Then for $z \in$ $\bar{D}\left(z_{0}, R_{1}\right)$, we have:

$$
\left|f^{(k)}(z)\right| \leq \frac{k ! R}{\left(R-R_{1}\right)^{k+1}}\|f\|_{R} .
$$

Proof. This lemma is an application of Cauchy's integral formula. For details [Lan99a], pp. 131.

Proof of lemma 3.4.

1. $\frac{d}{d z}$ is linear.

2. To prove tameness we use lemma 3.5. Let $z \in A_{n}$ and choose $R=e^{-(n+1)}(e-$ 1). Thus $\bar{D}(z, R) \subset A_{n+1}$. Hence $\|f\|_{R} \leq\|f\|_{n+1}$. 
In the notation of lemma 3.5 we can use that $R_{1}=0$ and $k=1$ and calculate in this way:

$$
\left\|f^{\prime}(z)\right\|_{n} \leq \frac{R}{R^{2}}\|f\|_{n+1}=\frac{e^{n+1}}{e-1}\|f\|_{n+1} .
$$

This description is independent of $z$. Thus

$$
\left\|f^{\prime}\right\|_{n} \leq \frac{e^{n+1}}{e-1}\|f\|_{n+1} .
$$

Thus the differential is $\left(1,0, \frac{e^{n+1}}{e-1}\right)$-tame.

A further class of spaces that is important for the description of twisted KacMoody algebras (compare definition 4.6) are spaces of holomorphic functions that satisfy some functional equation. We describe first the general setting and specialize then to the two most important cases, namely symmetric and antisymmetric holomorphic functions.

Lemma 3.6 (Subspaces of $\left.\operatorname{Hol}\left(\mathbb{C}^{*}, \mathbb{C}\right)\right)$. Let $k, l \in \mathbb{N}$ and $\omega=e^{\frac{2 \pi i}{k}}$. The spaces

$$
\operatorname{Hol}^{k, l}\left(\mathbb{C}^{*}, \mathbb{C}\right):=\left\{f \in \operatorname{Hol}\left(\mathbb{C}^{*}, \mathbb{C}\right) \mid f(\omega z)=\omega^{l} f(z)\right\}
$$

are tame Fréchet spaces.

Proof. As usual for $f \in \operatorname{Hol}^{k, l}\left(\mathbb{C}^{*}, \mathbb{C}\right)$, we put $\|f\|_{n}:=\sup _{z \in A_{n}}|f(z)|$. As $\mathrm{Hol}^{k, l}\left(\mathbb{C}^{*}, \mathbb{C}\right)$ is a closed subspace of $\operatorname{Hol}\left(\mathbb{C}^{*}, \mathbb{C}\right)$, it is a tame Fréchet space as a consequence of lemma 2.15 .

Twisted affine Kac-Moody algebras arise as fixed point algebras of diagram automorphisms of non-twisted affine Kac-Moody algebras. The list of possible diagram automorphisms shows that nontrivial diagram automorphisms have order $k=2$ or $k=3$ [Car02]. Thus the values of $k$ which are important for us are $k=2$ and $k=3$. For $k=2$, lemma 3.6 has the corollaries:

Corollary 3.7 (symmetric and antisymmetric loops).

- The space $\operatorname{Hol}^{s}\left(\mathbb{C}^{*}, \mathbb{C}\right):=\left\{f \in \operatorname{Hol}\left(\mathbb{C}^{*}, \mathbb{C}\right), f(z)=f(-z)\right\}$ is a tame Fréchet space.

- The space $\operatorname{Hol}^{a}\left(\mathbb{C}^{*}, \mathbb{C}\right):=\left\{f \in \operatorname{Hol}\left(\mathbb{C}^{*}, \mathbb{C}\right), f(z)=-f(-z)\right\}$ is a tame Fréchet space.

Lemmata 2.15 and 3.3 and corollary 3.7 include the following result:

Corollary 3.8. $F:=\operatorname{Hol}\left(\mathbb{C}^{*}, \mathbb{C}^{n}\right), F^{s}:=\operatorname{Hol}^{s}\left(\mathbb{C}^{*}, \mathbb{C}^{n}\right)$ and $F^{a}:=\operatorname{Hol}^{a}\left(\mathbb{C}^{*}, \mathbb{C}^{n}\right)$ are tame Fréchet spaces.

Let $V^{n}$ be a $n$-dimensional complex vector space. We want a tame structure on $\operatorname{Hol}\left(\mathbb{C}^{*}, V^{n}\right)$. Using the corollary 3.8 we get a tame Fréchet structure on the space $F:=\operatorname{Hol}\left(\mathbb{C}^{*}, \mathbb{C}^{n}\right)$. This yields a tame structure on the spaces $\operatorname{Hol}\left(\mathbb{C}^{*}, V^{n}\right)$, $\operatorname{Hol}^{s}\left(\mathbb{C}^{*}, V^{n}\right)$ and $\mathrm{Hol}^{a}\left(\mathbb{C}^{*}, V^{n}\right)$ only after the choice of an identification of $V^{n}$ with $\mathbb{C}^{n}$, hence a choice of a basis. As this construction uses this identification of $V^{n}$ with $\mathbb{C}^{n}$ we have to prove that the resulting tame structure is independent of it.

This is the content of the following lemma: 
LEMMA 3.9. Let $V^{n}$ be a n-dimensional complex vector space equipped with two norms $|$.$| and |\cdot|^{\prime}$. Study the spaces $\operatorname{Hol}^{k, l}\left(\mathbb{C}^{*}, V^{n}\right)$. Define gradings $\|f\|_{n}:=$ $\sup _{z \in A_{n}}|f(z)|$ and $\|f\|_{n}^{\prime}:=\sup _{z \in A_{n}}|f(z)|^{\prime}$. Those gradings are tame equivalent.

Proof. Any two norms on a finite dimensional vector space are equivalent (see any book about elementary analysis, i.e. [Kö0]). Thus there exist constants $c_{1}$ and $c_{2}$ such that $|x| \leq c_{1}|x|^{\prime}$ and $|x|^{\prime} \leq c_{2}|x|$. Then $\|f\|_{n}:=\sup _{z \in A_{n}}|f(z)| \leq \sup _{z \in A_{n}} c_{1}|f(z)|^{\prime}=c_{1}\|f\|_{n}^{\prime}$ and $\|f\|_{n}^{\prime}:=\sup _{z \in A_{n}}|f(z)|^{\prime} \leq \sup _{z \in A_{n}} c_{2}|f(z)|^{\prime}=c_{2}\|f\|_{n}$. Thus they are tame equivalent. $\square$

Corollary 3.10. Any identification of $\varphi: \mathbb{C}^{n} \longrightarrow V^{n}$ yields a 1-norm $\|v\|=$ $\sum v_{i}$ on $V^{n}$. The tame structures induced on $\operatorname{Hol}\left(\mathbb{C}^{*}, \mathbb{C}\right)$ by two different choices are equivalent. Hence the tame structure on $V^{n}$ is independent of the identification of $V^{n}$ with $\mathbb{C}^{n}$.

As a consequence we have established the following result:

TheOREm 3.11. Let $V^{n}$ be a complex vector space and \|\| any norm on $V^{n}$. The space $\operatorname{Hol}\left(\mathbb{C}^{*}, V^{n}\right)$ with the family of norms

$$
\|f\|_{n}=\sup _{z \in A_{n}}\|f(z)\|
$$

is a tame Fréchet space.

Corollary 3.12. Let $V^{n}$ be a complex vector space and ||$_{2}$ the Euclidean norm on $V^{n}$. The space $\operatorname{Hol}\left(\mathbb{C}^{*}, V^{n}\right)$ with the family of norms

$$
\|f\|_{n}=\sup _{z \in A_{n}}|f(z)|_{2}
$$

is a tame Fréchet space.

4. Tame structures on loop algebras and loop groups. In this chapter we introduce the functional analytic setting, which we use for the construction of affine Kac-Moody symmetric spaces in [Fre13a]: We define Kac-Moody groups and Kac-Moody algebras of holomorphic loops and prove that they are tame Fréchet Lie groups resp. Lie algebras. Via the loop realization Kac-Moody groups (resp. algebras) can be viewed as 2-dimensional extensions of loop groups. From a functional analytic point of view, a 2-dimensional extension is unproblematic; the only exception is, that the action of group elements on the two dimensional extension has to be well-defined as well. Nevertheless remark that this extension plays a crucial role for geometric considerations. In consequence we focus our attention on the loop group (resp. algebra). In subsection 4.2 we describe the loop algebras, in subsection 4.3 we turn our attention to the loop groups.

4.1. Affine Kac-Moody algebras. In this section we recall some basic facts about affine Kac-Moody algebras and introduce the more general notion of geometric affine Kac-Moody algebras. A thorough study of the algebraic properties of geometric affine Kac-Moody algebras is done in [Fre13c]. The loop algebra realization of (algebraic) affine Kac-Moody algebras is developed in the books [Kac90] and [Car02] from an algebraic point of view. We follow the more geometric approach to loop algebra realizations of Kac-Moody algebras, using the notion of [Ter95, HG09]. 
Let $\mathfrak{g}$ be a finite dimensional reductive Lie algebra over $\mathbb{F}=\mathbb{R}$ or $\mathbb{C}$. Hence by definition $\mathfrak{g}=\mathfrak{g}_{s} \oplus \mathfrak{g}_{a}$ is a direct product of a semisimple Lie algebra $\mathfrak{g}_{s}$ with an Abelian Lie algebra $\mathfrak{g}_{a}$. Let furthermore $\sigma=\left(\sigma_{s}, \sigma_{a}\right)$ be some involution of $\mathfrak{g}$, such that $\sigma_{s} \in \operatorname{Aut}\left(\mathfrak{g}_{s}\right)$ denotes an automorphism of finite order of $\mathfrak{g}_{s}$ such that the restriction of $\sigma$ to any simple factor $\mathfrak{g}_{i}$ of $\mathfrak{g}$ is an automorphism of $\mathfrak{g}_{i}$ and $\sigma_{a}=\left.\sigma\right|_{\mathfrak{g}_{a}}=$ Id. If $\mathfrak{g}_{s}$ is a Lie algebra over $\mathbb{R}$ we assume $\mathfrak{g}_{s}$ to be a Lie algebra of compact type. We define the loop algebra $L(\mathfrak{g}, \sigma)$ as follows

$$
L(\mathfrak{g}, \sigma):=\{f: \mathbb{R} \longrightarrow \mathfrak{g} \mid f(t+2 \pi)=\sigma f(t), f \text { satisfies some regularity conditions }\} \text {. }
$$

We use the notation $L(\mathfrak{g}, \sigma)$ to describe in a unified way constructions that can be realized explicitly for loop algebras satisfying various regularity conditions. Regularity conditions used in applications include the following:

- $H^{0}$-Sobolev loops, denoted $L^{0} \mathfrak{g}$,

- smooth, denoted $L^{\infty} \mathfrak{g}$,

- real analytic, denoted $L_{\text {an }} \mathfrak{g}$,

- (after complexification of the domain of definition) holomorphic on $\mathbb{C}^{*}$, denoted $M \mathfrak{g}$,

- holomorphic on an annulus $A_{n} \subset \mathbb{C}$, denoted $A_{n} \mathfrak{g}$, or

- algebraic (or equivalently: with a finite Fourier expansion), denoted $L_{\text {alg }} \mathfrak{g}$.

Definition 4.1 (affine Kac-Moody algebra). The indecomposable geometric affine Kac-Moody algebra associated to a pair $(\mathfrak{g}, \sigma)$ as described above is the algebra:

$$
\widehat{L}(\mathfrak{g}, \sigma):=L(\mathfrak{g}, \sigma) \oplus \mathbb{F} c \oplus \mathbb{F} d,
$$

equipped with the lie bracket defined by:

$$
\begin{aligned}
{[d, f(t)] } & :=f^{\prime}(t), \\
{[c, c]=[d, d] } & :=0, \\
{[c, d]=[c, f(t)] } & :=0, \\
{[f, g](t) } & :=[f(t), g(t)]_{0}+\omega(f(t), g(t)) c .
\end{aligned}
$$

Here $f \in L(\mathfrak{g}, \sigma)$ and $\omega$ is a certain antisymmetric 2-form on $M \mathfrak{g}$, satisfying the cocycle condition. If the regularity of $L(\mathfrak{g}, \sigma)$ is chosen such that $L(\mathfrak{g}, \sigma)$ contains non-differentiable functions, then $d$ is defined on the (dense) subspace of differentiable functions.

Explicit realizations using different regularity conditions are common. We use holomorphic or algebraic loops; for these we can define

$$
\omega(f, g):=\operatorname{Res}\left(\left\langle f, g^{\prime}\right\rangle\right) .
$$

Let us reformulate the definition for non twisted affine Kac-Moody algebras in terms of functions on $\mathbb{C}^{*}$ : Assume $\sigma=\mathrm{Id}$. First we develop a function $f \in L(\mathfrak{g}, \mathrm{Id})$ into its Fourier series $f(t)=\sum a_{n} e^{i n t}$. Then this function is naturally defined on a circle $S^{1}$; we understand this circle to be embedded as the unit circle $\left\{z \in \mathbb{C}^{*}|| z \mid=1\right\} \subset \mathbb{C}^{*}$; in this way the parameter $t$ gets replaced by the complex parameter $z:=e^{i t}$, with $|z|=1$; understanding the Fourier expansion now as a Laurent expansion of $F$, we can calculate the annulus, $A_{n}$, on which this series is defined. For example the holomorphic realization $M \mathfrak{g}$, is defined by the condition, that for any $f \in M \mathfrak{g}$ the 
Fourier expansion describes a Laurent series expansion of a holomorphic function on $\mathbb{C}^{*}$.

Definition 4.2. The complex geometric affine Kac-Moody algebra associated to a pair $(\mathfrak{g}, \sigma)$ is the algebra

$$
\widehat{M \mathfrak{g}}:=M \mathfrak{g} \oplus \mathbb{C} c \oplus \mathbb{C} d,
$$

equipped with the lie bracket defined by:

$$
\begin{aligned}
{[d, f(z)] } & :=i z f^{\prime}(z), \\
{[c, c]=[d, d] } & :=0 \\
{[c, d]=[c, f(z)] } & :=0 \\
{[f, g](z) } & :=[f(z), g(z)]_{0}+\omega(f(z), g(z)) c .
\end{aligned}
$$

As $\frac{d}{d t} e^{i t n}=i n e^{i t n}=i n z^{n}=i z \frac{d}{d z} z^{n}$ both definitions coincide.

Definition 4.3 (semisimple geometric affine Kac-Moody algebra).

A geometric affine Kac-Moody algebra $\widehat{L}(\mathfrak{g}, \sigma)$ is called

- semisimple if $\mathfrak{g}$ is semisimple,

- simple if $\mathfrak{g}$ is simple.

4.2. Lie algebras of holomorphic maps. Let $\mathfrak{g}$ be a complex reductive Lie algebra that is a direct product of simple Lie algebras with an Abelian Lie algebra. Simple Lie algebras are completely classified by their root systems; a complete list is given as follows:

$$
A_{n}, B_{n, n \geq 2}, C_{n, n \geq 3}, D_{n, n \geq 4}, E_{6}, E_{7}, E_{8}, F_{4}, G_{2} .
$$

Abelian Lie algebras are classified by their dimension. A complex reductive Lie algebra $\mathfrak{g}_{\mathbb{C}}$ has an up to conjugation unique compact real form $\mathfrak{g}_{c}$. This compact real form is defined as the real reductive Lie algebra $\mathfrak{g}_{\mathbb{R}}$ such that $\mathfrak{g}$ is a direct product of the (up to conjugation) unique compact real forms of the simple factors of $\mathfrak{g}_{\mathbb{C}}$ together with a compact real form of the Abelian factor. A compact real Abelian Lie algebra of dimension $n$ is just $\mathbb{R}^{n}$ together with the trivial bracket; but we define the exponential function such that the exponential image is a torus. Hence a compact real Lie algebra can be identified with the purely imaginary part of a complex Abelian Lie algebra.

DEFINITION 4.4 (complex holomorphic non-twisted Loop algebra). Let $\mathfrak{g}_{\mathbb{C}}$ be a finite-dimensional reductive complex Lie algebra.

1. The loop algebra $A_{n} \mathfrak{g}_{\mathbb{C}}$ is the vector space

$$
A_{n} \mathfrak{g}_{\mathbb{C}}:=\bigcup_{A_{n} \subset U \text { open }}\left\{f: U \longrightarrow \mathfrak{g}_{\mathbb{C}} \mid \mathrm{f} \text { is holomorphic }\right\},
$$

equipped with the natural Lie bracket:

$$
[f, g]_{L_{n} \mathfrak{g}}(z):=[f, g]_{0}(z):=[f(z), g(z)]_{\mathfrak{g}} .
$$

2. The loop algebra $M \mathfrak{g}_{\mathbb{C}}$ is the vector space

$$
M \mathfrak{g}_{\mathbb{C}}:=\left\{f: \mathbb{C}^{*} \longrightarrow \mathfrak{g}_{\mathbb{C}} \mid \mathrm{f} \text { is holomorphic }\right\},
$$

equipped with the natural Lie bracket:

$$
[f, g]_{M \mathfrak{g}}(z):=[f, g]_{0}(z):=[f(z), g(z)]_{\mathfrak{g}} .
$$




\section{LEMMA 4.5 .}

1. $M \mathfrak{g}_{\mathbb{C}}$ is a tame space.

2. $A_{n} \mathfrak{g}_{\mathbb{C}}$ is a Banach space.

Proof. The first assertion is a consequence of corollary 3.8. The second assertion is a consequence of Montel's theorem stating that absolute convergent sequences of holomorphic functions converge to a holomorphic function [BG91].

The inclusions $S^{1}=A_{0} \subset \ldots A_{n} \subset A_{n+1} \subset \cdots \subset \mathbb{C}^{*}$ induce the reversed inclusions on the associated loop algebras:

$$
M \mathfrak{g}_{\mathbb{C}} \subset \cdots \subset A_{n+1} \mathfrak{g}_{\mathbb{C}} \subset A_{n} \mathfrak{g}_{\mathbb{C}} \subset \cdots \subset A_{0} \mathfrak{g}_{\mathbb{C}}=L_{\mathrm{hol}} \mathfrak{g}_{\mathbb{C}}
$$

$L_{\mathrm{hol}} \mathfrak{g}_{\mathbb{C}}$ denotes functions holomorphic in a small open neighborhood around $S^{1} \subset$ $\mathbb{C}^{*}$.

To describe the twisted loop algebras we recall the graph automorphisms of the finite dimensional simple Lie algebras: the following list contains the simple algebras $A$ with a nontrivial diagram automorphism $\sigma$ and the type of the fixed point algebra (compare [Car02]).

$$
\begin{array}{lcccccc}
A & : & A_{2 k} & A_{2 k+1} & D_{k+1} & D_{4} & E_{6} \\
\text { Order of } \sigma & : & 2 & 2 & 2 & 3 & 2 \\
A^{1} & : & B_{k} & C_{k} & B_{k} & G_{2} & F_{4}
\end{array}
$$

Definition 4.6 ((twisted) loop algebra, $\operatorname{ord}(\sigma)=2$ ). Let $\mathfrak{g}_{\mathbb{C}}$ be a finite dimensional semisimple complex Lie algebra of type $A_{k}, D_{k, k \geq 5}$ or $E_{6}, \sigma$ the diagram automorphism. Let $\mathfrak{g}_{\mathbb{C}}:=\mathfrak{g}_{\mathbb{C}}^{1} \oplus \mathfrak{g}_{\mathbb{C}}^{-1}$ be the decomposition into the \pm -eigenspaces of $\sigma$. Let $X \in\left\{A_{n}, \mathbb{C}^{*}\right\}$. If $X=A_{n}$ holomorphic functions on $X$ are understood to be holomorphic on an open set containing $X$.

Then the loop algebra $(X \mathfrak{g})^{\sigma}$ is the vector space

$$
X \mathfrak{g}^{\sigma}:=\{f \in X \mathfrak{g} \mid f(-z)=\sigma(f(z))\},
$$

equipped with the natural Lie bracket:

$$
[f, g]_{X \mathfrak{g}^{\sigma}}(z):=[f, g]_{0}(z):=[f(z), g(z)]_{\mathfrak{g}} .
$$

REMARK 4.7 ((twisted) loop algebra, ord $(\sigma)=3$ ). For the algebra of type $D_{4}$ there exists an automorphism $\sigma$ of order 3 . In this case we get exactly the same results as for the other types. The main difference is that we have three eigenspaces, corresponding to $\left\{\omega, \omega^{2}, \omega^{3}=1\right\}$ for $\omega=e^{\frac{2 \pi i}{3}}$. For a function $f$ in the loop algebra $M \mathfrak{g}$, this results in a twisting condition $f(\omega z)=\sigma f(z)$ (for details compare again [Car02]).

Lemma 4.8 (Banach- and Fréchet structures on twisted loop algebras).

1. $A_{n} \mathfrak{g}^{\sigma}$ equipped with the norm \|\|$_{n}$ is a Banach Lie algebra,

2. $M \mathfrak{g}^{\sigma}$ equipped with the norms \|\|$_{n}$ is a tame Fréchet Lie algebra.

Proof. Closed subspaces of Banach spaces are Banach spaces and closed subspaces of tame Fréchet spaces are tame Fréchet spaces (lemma 2.15).

Recall the following definitions 
Definition 4.9 (compact-open topology). Let $\left(X, \mathcal{O}_{X}\right)$ and $\left(Y, \mathcal{O}_{Y}\right)$ be topological spaces and let $C(X, Y)$ denote the space of continuous functions $f: X \rightarrow Y$. The compact-open topology on $C(X, Y)$ is the topology generated by the subbasis consisting of all open neighborhoods $\mathcal{U} K, O$ defined by

$$
\mathcal{U} K, O:=\{f \in C(X, Y) \mid f(K) \subset O\} .
$$

Definition 4.10 (topology of compact convergence). Let $\left(X, \mathcal{O}_{X}\right)$ be a topological space and $\left(Y, d_{Y}\right)$ be a metric space. For any $\epsilon>0$, any function $f \in C(X, Y)$ and any compact set $K \subseteq X$ define the open ball $B_{\epsilon}^{K}(f)$ by

$$
B_{\epsilon}^{K}(f):=\{g \in C(X, Y) \mid d(f(x), g(x)) \leq \epsilon \forall x \in K\} .
$$

The topology of compact convergence on $C(X, Y)$ is the topology generated by the subbasis consisting of all open neighborhoods $B_{\epsilon}^{K}(f)$.

THEOREM 4.11. The following topologies on $M \mathfrak{g}_{\mathbb{C}}$ are equivalent:

1. the compact-open topology,

2. the topology of compact convergence,

3. the Fréchet topology.

Recall that on a Fréchet space $\left(F,\|\|_{n}\right)$

$$
d(f, g)=\sum_{n} \frac{1}{2^{n}} \frac{\|f-g\|_{n}}{1+\|f-g\|_{n}}
$$

defines a metric. The Fréchet topology coincides with the topology generated by the metric $d(f, g)$ (see [Ham82], chapter I.1).

Proof. We prove $(1) \Leftrightarrow(2),(3) \Leftrightarrow(2)$.

$(1) \Leftrightarrow(2)$ The equivalence between the compact-open topology and the topology of compact convergence is well-known for spaces of continuous functions $C(X, Y)$ for $X, Y$ metric spaces (see for example [Str06], p.96). In our case $X=\mathbb{C}^{*}$ and $Y=\mathfrak{g}$ are complex spaces. The extension to the setting of holomorphic functions is a straightforward consequence of Montel's theorem: Assuming $\left(f_{n}\right) \in \operatorname{Hol}\left(\mathbb{C}^{*}, \mathfrak{g}\right) \subset C\left(\mathbb{C}^{*}, \mathfrak{g}\right)$. Then by Montels theorem, the limit function $f=\lim _{n \rightarrow \infty} f_{n}$ is in $\operatorname{Hol}\left(\mathbb{C}^{*}, \mathfrak{g}\right)$ as well.

$(3) \Leftrightarrow(2)$ Let $\left(f_{k}\right) \subset M \mathfrak{g}$ be a sequence converging to $f_{0}$ in the topology of compact convergence. Then for every $n_{0} \in \mathbb{N}$ and $\epsilon>0$ there is a $k_{0}$ such that for all $k \geq k_{0}$ the estimate $\left\|f_{k}-f_{0}\right\|_{n} \leq \epsilon$ is satisfied for all $n \leq n_{0}$. Hence

$$
\begin{aligned}
d\left(f_{k}, f_{0}\right) & =\sum_{n=0}^{\infty} \frac{1}{2^{n}} \frac{\left\|f_{k}-f_{0}\right\|_{n}}{1+\left\|f_{k}-f_{0}\right\|_{n}}= \\
& =\sum_{n=0}^{n_{0}} \frac{1}{2^{n}} \frac{\left\|f_{k}-f_{0}\right\|_{n}}{1+\left\|f_{k}-f_{0}\right\|_{n}}+\sum_{n=n_{0}+1}^{\infty} \frac{1}{2^{n}} \frac{\left\|f_{k}-f_{0}\right\|_{n}}{1+\left\|f_{k}-f_{0}\right\|_{n}} \leq \\
& \leq \sum_{n=0}^{n_{0}} \frac{1}{2^{n}} \frac{\epsilon}{1+\epsilon}+\sum_{n=n_{0}+1}^{\infty} \frac{1}{2^{n}} \leq \\
& \leq 2 \epsilon+\left(\frac{1}{2}\right)^{n_{0}} .
\end{aligned}
$$


Hence $\left(f_{k}\right) \subset M \mathfrak{g}$ converges in the Fréchet topology. Conversely let $\left(f_{k}\right) \subset$ $M \mathfrak{g}$ be a sequence converging to $f_{0}$ in the Fréchet topology. Then we have

$$
\lim _{k \rightarrow \infty} d\left(f_{k}, f_{0}\right)=\lim _{k \rightarrow \infty} \sum_{n} \frac{1}{2^{n}} \frac{\left\|f_{k}-f_{0}\right\|_{n}}{1+\left\|f_{f}-f_{0}\right\|_{n}}=0 .
$$

As $d\left(f_{k}, f_{0}\right) \geq \sup _{n} \frac{1}{2^{n}} \frac{\left\|f_{k}-f_{0}\right\|_{n}}{1+\left\|f_{f}-f_{0}\right\|_{n}}$ we conclude that

$$
\lim _{k \rightarrow \infty} \sup _{n} \frac{1}{2^{n}} \frac{\left\|f_{k}-f_{0}\right\|_{n}}{1+\left\|f_{f}-f_{0}\right\|_{n}}=0 .
$$

As for any compact set $K \subset \mathbb{C}^{*}$ there is some $n$ such that $K \subset A_{n}$, this yields compact convergence.

The adjoint action $a d(g): M \mathfrak{g}^{\sigma} \longrightarrow M \mathfrak{g}^{\sigma}$ is $\left(0,0,2\|g\|_{n}\right)$-tame for each $g \in M \mathfrak{g}^{\sigma}$. Contrast this with the situation for affine Kac-Moody algebras described in section 6 . Having described the holomorphic complex loop algebras which we need, we turn now to some objects derived from them, namely the compact real forms and spaces of differential forms.

We start with real forms of compact type:

DEFINITION 4.12 (compact real form of a holomorphic non-twisted loop algebra). Let $\mathfrak{g}_{\mathbb{C}}$ be a finite-dimensional semisimple complex Lie algebra and $\mathfrak{g}$ its compact real form. The loop algebra $X \mathfrak{g}_{\mathbb{R}}^{\sigma}$ is the vector space

$$
X \mathfrak{g}_{\mathbb{R}}^{\sigma}:=\left\{f \in X \mathfrak{g}_{\mathbb{C}}^{\sigma} \mid f\left(S^{1}\right) \subset \mathfrak{g}\right\}
$$

equipped with the natural Lie bracket:

$$
[f, g]_{X \mathfrak{g}}(z):=[f, g]_{0}(z):=[f(z), g(z)]_{\mathfrak{g}} .
$$

As a holomorphic function on $X$ can be expanded into its Laurent series, one can represent every element of a loop algebra by a series

$$
f(z):=\sum_{n} g_{n} z^{n}
$$

with $g_{n} \in \mathfrak{g}$. $-\bar{g}_{-n}^{t}$.

LEMmA 4.13. The condition $f\left(S^{1}\right) \subset \mathfrak{g}_{\mathbb{R}}$ is equivalent to the condition $g_{n}=$

Proof. Let $z=e^{i t} \in S^{1} \subset \mathbb{C}^{*}$ and let $g_{n}=g_{n}^{r}+i g_{n}^{i}$ be the decomposition of $g_{n}$ into its real and imaginary parts. Then we find

$$
\begin{aligned}
f(z)= & \sum_{n \in \mathbb{Z}} g_{n} z^{n}=\sum_{n \in \mathbb{Z}} g_{n} e^{i t n}= \\
= & a_{0}+\sum_{n \in \mathbb{N}} g_{n} e^{i t n}+g_{-n} e^{-i t n}= \\
= & a_{0}+\sum_{n \in \mathbb{N}}\left(g_{n}^{r}+i g_{n}^{i}\right)(\cos (t n)+i \sin (t n))+\left(g_{-n}^{r}+i g_{-n}^{i}\right)(\cos (-t n)+i \sin (-t n))= \\
= & a_{0}+\sum_{n \in \mathbb{N}}\left(g_{n}^{r} \cos (t n)-g_{n}^{i} \sin (t n)\right)+i\left(g_{n}^{i} \cos (t n)+g_{n}^{r} \sin (t n)\right)+ \\
& \left(g_{-n}^{r} \cos (-t n)-g_{-n}^{i} \sin (-t n)+i\left(g_{-n}^{i} \cos (-t n)+\left(g_{-n}^{r} \sin (-t n)\right)=\right.\right. \\
= & a_{0}+\sum_{n \in \mathbb{N}}\left(g_{n}^{r}+g_{-n}^{r}+i\left(g_{n}^{i}+g_{-n}^{i}\right)\right) \cos (t n)+\left(-g_{n}^{i}+g_{-n}^{i}+i\left(g_{n}^{r}-g_{-n}^{r}\right)\right) \sin (t n)
\end{aligned}
$$


Now $x \in \mathfrak{g}_{\mathbb{C}}$ is in $\mathfrak{g}$ if $x=-\bar{x}^{t}$. This gives for the coefficients $g_{n}^{r}$ :

$$
\begin{aligned}
& g_{n}^{r}+g_{-n}^{r}=-\left(g_{n}^{r}\right)^{t}-\left(g_{-n}^{r}\right)^{t}, \\
& g_{n}^{r}-g_{-n}^{r}=\left(g_{n}^{r}\right)^{t}-\left(g_{-n}^{r}\right)^{t} .
\end{aligned}
$$

Adding both we get $g_{n}^{r}=-\left(g_{-n}^{r}\right)^{t}$. In a similar way we get for the imaginary parts of the coefficients $g_{n}^{i}=\left(g_{-n}^{i}\right)^{t}$ and thus the result.

LEMMA 4.14. $M \mathfrak{g}_{\mathbb{R}}$ is a tame Fréchet space.

Proof. $M \mathfrak{g}_{\mathbb{R}} \subset M \mathfrak{g}_{\mathbb{C}}$ is a closed subspace and thus tame according to lemma 2.15.

\section{DEFINITION 4.15.}

1. $\Omega^{1}\left(X, \mathfrak{g}_{\mathbb{C}}\right)$ is the space of $\mathfrak{g}_{\mathbb{C}}$-valued 1 -forms on $X$; elements $\omega \in \Omega^{1}\left(X, \mathfrak{g}_{\mathbb{C}}\right)$ are of the form $\omega(z)=f(z) d z$ with $f(z) \in X \mathfrak{g}_{\mathbb{C}}$. We define a family of norms by $\|\omega\|_{n}:=|f(z)|_{n}$.

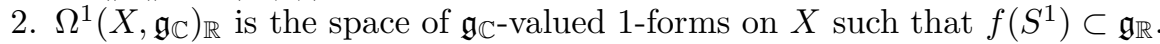

As $M \mathfrak{g}_{\mathbb{C}}$ and $M \mathfrak{g}_{\mathbb{R}}$ are tame Fréchet spaces, also $\Omega^{1}\left(X, \mathfrak{g}_{\mathbb{C}}\right)$ and $\Omega^{1}\left(X, \mathfrak{g}_{\mathbb{C}}\right)_{\mathbb{R}}$ are tame. Remark, that this is not the topology as a dual space.

REMARK 4.16. Real forms of the algebras $X \mathfrak{g}_{\mathbb{C}}^{\sigma}$ correspond to conjugate-linear involutions of $X \mathfrak{g}_{\mathbb{C}}^{\sigma}$ : assign to a real form the conjugation with respect to it. In the other direction, fixed point algebras of conjugate-linear involutions are real forms. Hence, real forms are closed subalgebras. Thus by an application of lemma 2.15 real forms of $M \mathfrak{g}_{\mathbb{C}}^{\sigma}$ are tame, real forms of $A_{n} \mathfrak{g}_{\mathbb{C}}^{\sigma}$ are Banach.

4.3. Lie groups of holomorphic maps. Up to now we studied analytic structures on loop algebras but not on the associated loop groups. In short the main result is that all loop algebras interesting to us are tame Lie algebras. In this section we prove similar results for loop groups. Let $G$ be a compact semisimple Lie group and $G_{\mathbb{C}}$ its complexification.

4.3.1. Foundations. Let us recall the definition of a smooth tame Lie group from [Ham82]:

Definition 4.17 (smooth tame Lie group). A smooth tame Lie group is a smooth tame Fréchet manifold $G$ equipped with a group structure such that the multiplication map

$$
\varphi: G \times G \longrightarrow G, \quad(g, h) \mapsto g h
$$

and the inverse map

$$
\varphi: G \longrightarrow G, \quad g \mapsto g^{-1}
$$

are smooth tame maps.

In this section we show that the following groups are smooth tame Lie groups:

DeFinition 4.18 (complex loop groups).

1. The loop group $A_{n} G$ is the group

$$
A_{n} G_{\mathbb{C}}:=\left\{f: A_{n} \longrightarrow G_{\mathbb{C}} \mid \mathrm{f} \text { is holomorphic }\right\} .
$$

The multiplication is defined to be $f g(z):=f(z) g(z)$ for $f, g \in A_{n} G$. 
2. The loop group $M G$ is the group

$$
M G_{\mathbb{C}}:=\left\{f: \mathbb{C}^{*} \longrightarrow G_{\mathbb{C}} \mid \mathrm{f} \text { is holomorphic }\right\} .
$$

The multiplication is defined to be $(f g)(z):=f(z) g(z)$ for $f, g \in M G$.

Definition 4.19 (real form of the compact type).

1. The real form of the compact type $A_{n} G_{\mathbb{R}}$ is defined to be

$$
A_{n} G_{\mathbb{R}}:=\left\{f \in A_{n} G_{\mathbb{C}} \mid f\left(S^{1}\right) \subset G_{\mathbb{R}}\right\} .
$$

2. The real form of the compact type $M G_{\mathbb{R}}$ is defined to be

$$
M G_{\mathbb{R}}:=\left\{f \in M G_{\mathbb{C}} \mid f\left(S^{1}\right) \subset G_{\mathbb{R}}\right\} .
$$

There are exponential functions

$$
\begin{aligned}
A_{n} \exp : A_{n} \mathfrak{g} & \longrightarrow A_{n} G \text { and } \\
\operatorname{Mexp}: M \mathfrak{g} & \longrightarrow M G,
\end{aligned}
$$

defined pointwise using the group exponential function $\exp : \mathfrak{g} \rightarrow G$ :

$$
\begin{aligned}
(z) & :=\exp (f(z)), \\
{[(\operatorname{Mexp})(f)](z) } & :=\exp (f(z)) .
\end{aligned}
$$

Let us remark, that for any $z \in \mathbb{C}^{*}$ resp. $z \in A_{n}$, the curve $\gamma(t):=[(\operatorname{Mexp})(t$. $f)](z)$ resp. $\gamma(t):=\left[\left(A_{n} \exp \right)(t \cdot f)\right]$ defines 1-parameter subgroups.

REMARK 4.20. In contrast to algebraic affine Kac-Moody algebras, for holomorphic Kac-Moody algebras and their Kac-Moody groups, the exponential map is defined on the whole Lie algebra $M \mathfrak{g}$.

The next important object needed to describe the connection between the loop algebras and the loop groups is the definition of the Adjoint action Ad:

As usual it is defined pointwise using the Adjoint action of the Lie group $G_{\mathbb{K}}$, $\mathbb{K} \in\{\mathbb{R}, \mathbb{C}\}:$

$$
\begin{array}{ll}
\left(\operatorname{Ad}\left(A_{n} G\right)_{\mathbb{K}} \times A_{n} \mathfrak{g}_{\mathbb{K}}\right) \longrightarrow A_{n} \mathfrak{g}_{\mathbb{K}}, & (f, h) \mapsto f h f^{-1}, \\
\left(\operatorname{Ad}(M G)_{\mathbb{K}} \times M \mathfrak{g}_{\mathbb{K}}\right) \longrightarrow M \mathfrak{g}_{\mathbb{K}}, & (f, h) \mapsto f h f^{-1},
\end{array}
$$

where

$$
f h f^{-1}(z):=f(z) h(z) f^{-1}(z) \simeq \operatorname{Ad}(f(z))(h(z)) .
$$

For the Adjoint action of groups of the compact type to be well-defined we have to check, that the condition $f\left(S^{1}\right) \subset \mathfrak{g}_{\mathbb{R}}$ is preserved. This is a consequence of the adjoint action for finite dimensional compact Lie groups: for all $z \in S^{1}$ we have $f(z) \in G_{\mathbb{R}}$ and $h(z) \in \mathfrak{g}_{\mathbb{R}}$. Thus the condition $\operatorname{Ad}(f) h(z) \in \mathfrak{g}_{\mathbb{R}}$ is preserved pointwise.

Lemma 4.21. The exponential function and the Adjoint action satisfy the identity:

$$
A d \circ \exp X=e^{a d} X \quad \text { for } \quad X \in\left\{A_{n}, \mathbb{C}^{*}\right\}
$$


Proof. Applying the well-known identity for finite dimensional Lie algebras (resp. Lie groups) we get that the identity is valid pointwise.

We now investigate the functional analytic nature of the groups $A_{n} G$ and $M G$ : to fix some notation let $X \sigma$ denote by abuse of notation an involution of $X \mathfrak{g}$ resp. of $X G$. Let $X G_{D}$ denote a real form of non-compact type of $X G$, and denote by $\operatorname{Fix}(X \sigma)$ the fixed point group of an involution $X \sigma$.

E. Heintze and C. Groß [HG09] show that real forms of the non-compact type of a complex simple Kac-Moody algebra are in bijection with involutions of the compact real form (which is unique up to conjugation). Let $X \mathfrak{g}_{\mathbb{R}}$ be a compact real form with involution $X \sigma$. We denote by $X \mathfrak{g}_{D, \sigma}$ the real form of non-compact type associated to $X \sigma$.

Let us focus on a description of the groups $A_{n} G$ : as $A_{n}$ is compact we can follow the classical strategy to define manifold and Lie group structures. We start by defining a chart on an open set containing the identity with values in the Lie algebra via the exponential map; then we use left translation to construct an atlas of the whole group. This strategy yields the following basic results:

THEOREM 4.22 .

1. $A_{n} G_{\mathbb{R}}$ and $A_{n} G_{\mathbb{C}}$ are Banach-Lie groups.

2. Real forms $A_{n} G_{D}$ of non-compact type of $A_{n} G_{\mathbb{C}}$ are Banach-Lie groups.

3. Quotients $A_{n} G_{\mathbb{R}} / F i x\left(A_{n} \sigma\right)$ and $A_{n} G_{D, \sigma} /$ Fix $\left(A_{n} \sigma\right)$ are Banach manifolds.

For Banach-Lie groups and Banach manifolds, there exists a huge literature; for a classical introduction see for example [Pal68, Lan99b].

For the groups $M G$ themselves the theory is considerably more difficult. The crucial observation is the fact that the exponential map is in general no local diffeomorphism. Let us give an example of this strange phenomenon:

EXAmple $4.23(M S L(2, \mathbb{C}))$. We study the Lie group $S L(2, \mathbb{C})$. As is well known,

$$
\exp : \mathfrak{s l}(2, \mathbb{C}) \longrightarrow S L(2, \mathbb{C})
$$

is not surjective. For example elements $g \in S L(2, \mathbb{C})$ conjugate to the element

$$
\left(\begin{array}{cc}
-1 & 1 \\
0 & -1
\end{array}\right)
$$

are not in the image of $\exp (\mathfrak{s l}(2, \mathbb{C}))$ (see $[\mathrm{DK} 00])$.

We want to show that there exists a sequence of functions $f_{n} \in M S L(2, \mathbb{C})$ which converges to the identity element

$$
\operatorname{Id}(z):=\left(\begin{array}{ll}
1 & 0 \\
0 & 1
\end{array}\right)
$$

of $\operatorname{MSL}(2, \mathbb{C})$ in the compact-open-(tame Fréchet) topology but is not contained in the image of $\operatorname{Mexp}(M \mathfrak{s l}(2, \mathbb{C}))$. To this end, we have to construct the functions $f_{n}$ in a way that each one contains at least one point $z_{n} \in \mathbb{C}^{*}$ that are not in the image of $\exp (\mathfrak{s l}(2, \mathbb{C}))$. Thus let us define

$$
f_{n}(z)=\left(\begin{array}{cc}
e^{\pi z / n} & -i z / n \\
0 & e^{-\pi z / n}
\end{array}\right)
$$


Then

$$
f_{n}(i n)=\left(\begin{array}{cc}
-1 & 1 \\
0 & -1
\end{array}\right) .
$$

In consequence $f_{n}$ is not contained in $\Im(\operatorname{Mexp}(\mathfrak{s l}(2, \mathbb{C})))$ for any $n \in \mathbb{N}$. On the other hand, for any fixed $z_{0} \in \mathbb{C}^{*}$ we calculate the $\operatorname{limit}_{n \rightarrow \infty} f_{n}\left(z_{0}\right)$ to be

$$
\lim _{n \rightarrow \infty} f_{n}\left(z_{0}\right)=\left(\begin{array}{ll}
1 & 0 \\
0 & 1
\end{array}\right)=\text { Id . }
$$

Hence in the compact-open topology for every neighborhood $U_{k}$ of the identity there exist $n_{k} \in \mathbb{N}$ such that $\forall n \geq n_{k}: f_{n} \in U_{k}$; hence, in the compact open topology

$$
\lim _{n \rightarrow \infty} f_{n}=\mathrm{Id} .
$$

This observation concludes the proof that Mexp $: M \mathfrak{s l}(2, \mathbb{C}) \rightarrow M S L(2, \mathbb{C})$ is not a local diffeomorphism.

A direct consequence is, that the holomorphic loop groups $M G$ for $G$ semisimple are no locally exponential Lie groups in the sense of K.-H. Neeb [Nee06], that is Lie groups such that exp is a local diffeomorphism.

Hence, we have to find a way to define manifold structures on $M G$ without making use of the exponential maps. We start by describing some results about the relationship between $M G$ and $M \mathfrak{g}$. Then we show that loop groups satisfy the weaker axioms for pairs of exponential type introduced by Hideki Omori.

Definition 4.24. The tangential space $T_{p}(M G)$ is defined as the space of pathequivalence classes of smooth paths. For $i=1,2$, let $\epsilon_{i}>0$. Then two smooth curves

$$
\gamma_{i}:\left(-\epsilon_{i}, \epsilon_{i}\right) \times \mathbb{C}^{*} \longrightarrow G_{\mathbb{C}}, i=1,2
$$

are equivalent if there is some $\epsilon_{0}>0$ such that

$$
\left.\gamma_{1}\right|_{\left(-\epsilon_{0}, \epsilon_{0}\right) \times \mathbb{C}^{*}}=\left.\gamma_{2}\right|_{\left(-\epsilon_{0}, \epsilon_{0}\right) \times \mathbb{C}^{*}}
$$

By definition, a path depends holomorphically on the second factor and smoothly on the first factor. Let us remark, that one can consider weaker regularity conditions on the first factor (i.e. $C^{k}$-dependence); we will not pursue the study of these weaker regularity conditions any further. In contrast the holomorphic dependence on the second factor is derived from the regularity condition imposed on the (holomorphic) Kac-Moody group.

The relationship between $M \mathfrak{g}$ and $M G$ is described by the following three results (for the convenience of the reader, we first state the three results and postpone the proofs after the discussion):

Theorem 4.25 (Tangential space). Let $\mathfrak{g}$ be the Lie algebra of $G$. Then

$$
M \mathfrak{g}=T_{e}(M G) .
$$

Moreover the tangential space $T_{e}(M G)$ is isomorphic to the Lie algebra of leftinvariant vector fields on $M G$. 
While the tangential space of a loop group is the corresponding loop algebra, the exponential map behaves in general badly. More precisely, we have the following result:

THEOREM 4.26 (Loop groups whose exponential map is no local diffeomorphism). Let $G_{\mathbb{C}}$ be a complex semisimple Lie group.

$$
\text { Mexp : } M \mathfrak{g} \longrightarrow M G
$$

is not a local diffeomorphism.

This is in sharp contrast to the case of nilpotent Lie groups. For these groups we have the following result:

Proposition 4.27 (Loop groups whose exponential map is a local diffeomorphism). Let $G_{\mathbb{C}}$ be a complex Lie group such that its universal cover is biholomorphically equivalent to $\mathbb{C}^{n}$. Then its exponential map Mexp is a local diffeomorphism.

Corollary 4.28. Let $G$ be a complex Lie group. If $G$ is nilpotent (i.e. Abelian) then Mexp is a diffeomorphism.

Corollary 4.28 is a direct consequence of proposition 4.27, as Abelian and nilpotent Lie groups have $\mathbb{C}^{n}$ as their universal cover [Kna96], corollary 1.103 and [Var84], section 3.6.

Theorems 4.25 and 4.26 may seem contradictory at first, as they state, that

Lie group $\quad \longrightarrow \quad$ Lie algebra : Good behavior!

Lie algebra $\quad \longrightarrow \quad$ Lie group : Bad behavior!

Nevertheless, it is a typical for infinite dimensional systems in the following sense:

The step from the Lie group to the Lie algebra is linearization or differentiation; differentiation exists in very general frameworks; typically no subtle obstacles arise; in contrast the step from the Lie algebra to the Lie group is integration; integration is in general frameworks a very subtle procedure [KM97, BGN04, Ber08, Gro97]. This behavior seems contradictory as the usual intuition, sharpened at finite dimensional manifolds, tells us there should be a geodesic in every direction. From the point of view of classical analysis, one can describe the analytic basics of this situation as a consequence of a change of limits, arising because of the non-compactness of $\mathbb{C}^{*}$ :

- On the one hand, theorem 4.25 describes locally uniform convergence on each compact subset $K \subset \mathbb{C}^{*}$.

- Theorem 4.26 shows in contrast that globally on $\mathbb{C}^{*}$ convergence needs no longer be uniform.

We now give the proofs that we have omitted:

Proof of theorem 4.25.

1. We prove first the inclusion $M \mathfrak{g} \subset T_{e}(M G)$. Hence we have to show the following: let $\gamma(t):(-\epsilon, \epsilon) \rightarrow M G$ be a curve in $M G$ such that $\gamma(0)=e \epsilon$ $M G$. Then $\dot{\gamma}(0)$ is in $M \mathfrak{g}$. Using, that $M \mathfrak{g}$ is a loop algebra, we have an equivalent description of $\dot{\gamma}(0)$ as a function $\dot{\gamma}(0, z): \mathbb{C}^{*} \longrightarrow \mathfrak{g}$, describing explicitly the loop.

Let $z_{0} \in \mathbb{C}^{*}$ arbitrary but fixed. Then $\gamma_{z_{0}}(t)$, the evaluation of $\gamma(t)$ at $z_{0} \in \mathbb{C}^{*}$, is a curve in $G$ satisfying $\gamma_{z_{0}}(0)=e$. Thus, using that the finite dimensional 
exponential map exp $: \mathfrak{g} \longrightarrow G$ is a local diffeomorphism, we get that $\frac{d}{d t} \gamma_{z_{0}}(t)$ is an element in $\mathfrak{g}$. As $z_{0}$ is arbitrary we have constructed a function $w$ : $\mathbb{C}^{*} \longrightarrow \mathfrak{g}$, satisfying

$$
w(z)=\dot{\gamma}(0, z)
$$

we have now to prove that $w$ is holomorphic.

We use, that $\gamma(t, z)$ depends holomorphically on $z$. This is equivalent to

$$
\frac{d}{d \bar{z}} \gamma(t, z)=0
$$

Hence

$$
0=\frac{d}{d t} \frac{d}{d \bar{z}} \gamma(t, z)=\frac{d}{d \bar{z}}\left[\frac{d}{d t} \gamma(t, z)\right]
$$

where we may switch the derivatives because of smoothness. Hence $\frac{d}{d t} \gamma(t, z)$ is holomorphic.

This shows: $T_{e}(M G) \subset M \mathfrak{g}$.

2. The other direction is straight forward: let $\gamma \in M \mathfrak{g}$. Then $\operatorname{Mexp}(t \gamma)$ is a curve in $M G$ with tangential vector $\gamma$. So $M \mathfrak{g} \subset T_{e}(M G)$.

This completes the proof.

Proof of theorem 4.26. The proof of theorem 4.26 relies on the fact that Mexp is no local diffeomorphism for $S L(2, \mathbb{C})$. Let $\mathfrak{h} \simeq \mathfrak{s l}(2, \mathbb{C}) \subset \mathfrak{g}$ be a subalgebra of $\mathfrak{g}$. Then there is an $H=S L(2, \mathbb{C})$ subgroup in $G$, such that $\mathfrak{h}$ is the Lie algebra of $H$. See part VII.5 of the book [Kna96]. Study the subalgebra $M \mathfrak{h} \subset M \mathfrak{g}$ and the subgroup $M H \subset M G . M \mathfrak{h}$ can be identified with $T_{e}(M H)$. Moreover Mexp $: M \mathfrak{h} \subset M H$. But as example 4.23 shows, Mexp is no local diffeomorphism. This completes the proof.

The image of the exponential map consists of those loops whose image is completely contained in the image of the Lie group exponential map. We give further comments about this situation in the third part, dealing with geometric aspects [Fre13a], where we study the behavior of geodesics.

Proof of theorem 4.2\%. Let $\widetilde{G}$ be the universal cover of $G$; the exponential map $\exp : \mathfrak{g} \longrightarrow \widetilde{G}$ is a biholomorphic map. Thus concatenation with $\exp \left(\right.$ resp. $\exp ^{-1}$ ) induces a biholomorphic map between $M \mathfrak{g}$ and $M \widetilde{G}$. To get that Mexp : $M \mathfrak{g} \longrightarrow M G$ is a local diffeomorphism, one uses the fact that each loop in $\widetilde{M G}$ projects onto a loop in $M G$ and, conversely, each loop in $M G$ can be lifted to a loop in $M \widetilde{G}$, which is unique up to Deck transformation and thus locally unique. This proves that Mexp is a local diffeomorphism.

4.3.2. Manifold structures on groups of holomorphic maps. In this section we prove that the groups $M G_{\mathbb{R}}, M G_{\mathbb{C}}$, and various quotients are tame Fréchet manifolds.

ThEOREM 4.29. $M G_{\mathbb{C}}$ is a tame Fréchet manifold.

The idea of the proof is to use logarithmic derivatives, to get charts is quite common: for regular Lie groups it is developed in the book [KM97], chapters 38 and 40. Furthermore it is used by K.-H. Neeb [Nee06] to prove the following theorem [Nee06], theorem III.1.9.: 
Theorem 4.30 (Neeb). Let $\mathbb{F} \in\{\mathbb{R}, \mathbb{C}\}, G$ be a $\mathbb{F}$-Lie group and $M$ a finite dimensional, connected $\sigma$-compact $\mathbb{F}$-manifold. We endow the group $C_{\mathbb{F}}^{\infty}(M, G)$ with the compact open $C^{\infty}$-topology, turning it into a topological group. This topology is compatible with a Lie group structure if $\operatorname{dim}_{\mathbb{F}} M=1$ and $\pi_{1}(M)$ is finitely generated.

The main ingredients for the proof of Neeb's theorem are the use of logarithmic derivatives to define charts in $\Omega^{1}(M, \mathfrak{g})$, the space of $\mathfrak{g}$-valued 1 -forms on $M$ and Glöckners inverse function theorem [Glö07], to take care of the monodromy if $\pi_{1}(M)$ is nontrivial.

If $\mathbb{F}=\mathbb{C}$ we have the equivalence $C_{\mathbb{F}}^{\infty}(M, G) \simeq \operatorname{Hol}(M, G)$. Our situation is the special case $M=\mathbb{C}^{*}$. Hence, $\pi_{1}(M)=\mathbb{Z}$ is a finitely generated group. The compact open $C^{\infty}$-topology coincides for holomorphic maps with the topology of compact convergence. Thus Neeb's theorem tells us that $M G_{\mathbb{F}}, \mathbb{F} \in\{\mathbb{R}, \mathbb{C}\}$ are locally convex topological Lie groups.

Nevertheless, we do not get tame structures. Hence we have to prove the theorem completely new. Our presentation follows the proof of K.-H. Neeb for the locally convex case.

We need some definitions: $\alpha \in \Omega^{1}(M, \mathfrak{g})$ is called integrable iff there exists a function $f \in \operatorname{Hol}(M, G)$ such that $\delta(f):=f^{-1} d f=\alpha$. The uniqueness of solutions to linear differential equations shows that $\delta\left(f_{1}\right)=\delta\left(f_{2}\right)$ iff $f_{1}=g f_{2}$ for some $g \in G$.

The first step of the proof is the following lemma, whose statement and proof can be found in [KM97] and [Nee06]: morally it is a straight forward application of the monodromy principle for holomorphic Pfaffian systems, as described in the article [NY02].

Lemma 4.31. Let $M$ be a 1-dimensional complex manifold, $\alpha \in \Omega^{1}(M, \mathfrak{g})$.

1. $\alpha$ is locally integrable

2. If $M$ is connected, $M_{0} \in M$, then there exists a homomorphism

$$
\operatorname{per}_{\alpha}: \pi_{1}\left(M, m_{o}\right) \longrightarrow G
$$

that vanishes iff $\alpha$ is integrable.

Proof. Proof of theorem 4.29 We define the embedding:

$$
\begin{array}{ccc}
\varphi: M G_{\mathbb{C}} & \hookrightarrow & \Omega^{1}\left(\mathbb{C}^{*}, \mathfrak{g}_{\mathbb{C}}\right) \times G_{\mathbb{C}} \\
f & \mapsto & \left(\delta(f)=f^{-1} d f, f(1)\right)
\end{array}
$$

This embedding is injective as $\left(\delta\left(f_{1}\right), f_{1}(1)\right)=\left(\delta\left(f_{2}\right), f_{2}(1)\right)$ iff $\delta\left(f_{1}\right)=\delta\left(f_{2}\right)$ and $f_{1}(1)=f_{2}(1)$. Using the uniqueness of solutions (up to the starting point) of the linear differential equation $d f=f \omega$ where $\omega:=f_{1}^{-1} d f_{1}$, the first condition leads to the relation $f_{1}=g f_{2}$ for some $g \in G$. Then the second condition gives uniqueness as $g f_{2}(1)=f_{2}(1)$ leads to $g=\mathrm{Id}$.

Compare this embedding with the description of polar actions on Fréchet spaces in section 5 . Let $\pi_{1}$ and $\pi_{2}$ denote the projections:

$$
\begin{array}{lllc}
\pi_{1}: \Omega^{1}\left(\mathbb{C}^{*}, \mathfrak{g}_{\mathbb{C}}\right) \times G_{\mathbb{C}} & \mapsto & \Omega^{1}\left(\mathbb{C}^{*}, \mathfrak{g}_{\mathbb{C}}\right), \\
\pi_{2}: \Omega^{1}\left(\mathbb{C}^{*}, \mathfrak{g}_{\mathbb{C}}\right) \times G_{\mathbb{C}} & \mapsto & G_{\mathbb{C}}
\end{array}
$$

We construct charts for $M G_{\mathbb{C}} \subset \Omega^{1}\left(\mathbb{C}^{*}, \mathfrak{g}_{\mathbb{C}}\right) \times G_{\mathbb{C}}$ as direct product of charts for $\pi_{1} \circ \varphi\left(M G_{\mathbb{C}}\right)$ and $\pi_{2} \circ \varphi\left(M G_{\mathbb{C}}\right)$. 
- $\pi_{2} \circ \varphi$ is surjective; hence a describing of charts for the second factor can be done by choosing charts for $G$. Via the exponential mapping and left translation, we get charts $\psi_{2, g}: U(g) \longrightarrow V(0)$ defined on an open set $U(g)$ around $g \in G$ taking values in $V(0) \subset \mathfrak{g}_{\mathbb{C}}$. To describe the family of norms, we use the Euclidean metric

$$
\|\|_{n}:=\|\|_{E u c l} .
$$

- The first factor is more difficult to deal with as $\pi_{1} \circ \varphi$ is not surjective. While every $\mathfrak{g}_{\mathbb{C}^{-v a l u e d}} 1$-form $\alpha \in \Omega\left(\mathbb{C}^{*}, \mathfrak{g}_{\mathbb{C}}\right)$ is locally integrable by lemma 4.31 , the monodromy may prevent global integrability. A form $\alpha \in \Omega^{1}\left(\mathbb{C}^{*}, \mathfrak{g}_{\mathbb{C}}\right)$ is in the image of $\pi_{1} \circ \varphi$ iff its monodromy vanishes, that is iff

$$
e^{\int_{S^{1}} \alpha}=e \in G_{\mathbb{C}} .
$$

This is equivalent to the condition $\int_{S^{1}} \alpha=a_{-1}(\alpha) \subset \frac{1}{2 \pi i} \exp ^{-1}(e)$ where $a_{-1}(\alpha)$ denotes the $(-1)$-Laurent coefficient of the Laurent series of $\alpha=$ $f(z) d z$. Hence we get the characterization of $\Im\left(\pi_{1} \circ \varphi\right)$ as the inverse image of $e \in G_{\mathbb{C}}$ via the monodromy map.

Thus we have to show that this inverse image is a tame Fréchet manifold. To this end, we use composition with a chart $\psi: U \longrightarrow V$ for $e \in U \subset G$ with values in $G_{\mathbb{C}}$. This gives us a tame map $\Omega\left(\mathbb{C}^{*}, \mathfrak{g}_{\mathbb{C}}\right) \longrightarrow \mathfrak{g}_{\mathbb{C}}$. This map satisfies the assumptions of the following theorem, whose proof can be found in [Fre12c].

TheOREM 4.32. Let $F$ be a tame space and $\varphi: F \rightarrow \mathbb{R}^{n}$ a tame map. Let $g \in \mathbb{R}^{n}$ be a regular value for $\varphi$. Then $\varphi^{-1}(g)$ is a tame Fréchet submanifold of co-finite type.

Thus its inverse image is a tame Fréchet submanifold. This proves that $\pi_{1} \circ \varphi$ is a tame Fréchet submanifold.

Thus $M G_{\mathbb{C}}$ as a product of a tame Fréchet manifold with a Lie group is a tame Fréchet manifold. This completes the proof of theorem 4.29.

TheOREm 4.33. $M G_{\mathbb{C}}$ is a tame Fréchet Lie group.

Proof. From theorem 4.29 we know that $M G_{\mathbb{C}}$ is tame Fréchet manifold. Hence we have to check that

$$
\psi_{1}: M G_{\mathbb{C}} \times M G_{\mathbb{C}} \longrightarrow M G_{\mathbb{C}}, \quad(g, f) \mapsto g f,
$$

and

$$
\psi_{2}: M G_{\mathbb{C}} \longrightarrow M G_{\mathbb{C}}, \quad f \mapsto f^{-1}
$$

are tame Fréchet maps. Using the embedding

$$
\begin{array}{cccc}
\varphi: M G_{\mathbb{C}} & \hookrightarrow & \Omega^{1}\left(\mathbb{C}^{*}, \mathfrak{g}_{\mathbb{C}}\right) \times G_{\mathbb{C}}, \\
f & \mapsto & \left(f^{-1} d f, f(1)\right) .
\end{array}
$$

We get for $\psi_{1}$ the description

$$
\begin{aligned}
\psi_{1}: \Omega^{1}\left(\mathbb{C}^{*}, \mathfrak{g}_{\mathbb{C}}\right) \times \Omega^{1}\left(\mathbb{C}^{*}, \mathfrak{g}_{\mathbb{C}}\right) \times G_{\mathbb{C}} \times G_{\mathbb{C}} \longrightarrow \Omega^{1}\left(\mathbb{C}^{*}, \mathfrak{g}_{\mathbb{C}}\right) \times G_{\mathbb{C}} \\
\left(f^{-1} d f, g^{-1} d g, f(1), g(1)\right) \mapsto\left(g f^{-1} d f g^{-1}+g^{-1} d g, f(1) g(1)\right),
\end{aligned}
$$


which is smooth as a direct product of smooth tame maps.

Similarly we get for $\psi_{2}$ :

$$
\begin{aligned}
\psi_{1}: \Omega^{1}\left(\mathbb{C}^{*}, \mathfrak{g}_{\mathbb{C}}\right) \times G_{\mathbb{C}} & \longrightarrow \Omega^{1}\left(\mathbb{C}^{*}, \mathfrak{g}_{\mathbb{C}}\right) \times G_{\mathbb{C}} \\
\left(f^{-1} d f, f(1)\right) & \mapsto\left(f\left(f^{-1} d f\right) f^{-1}, f^{-1}(1)\right),
\end{aligned}
$$

which is again a smooth tame map.

We now investigate different classes of quotients of loop groups:

Proposition 4.34. $M G_{\mathbb{R}}$ is a tame Fréchet Lie group.

Proof. The proof is similar to the proof for $M G_{\mathbb{C}}$. We have only to take care of the reality condition $f\left(S^{1}\right) \subset G_{\mathbb{R}}$ for loops $f \in M G_{\mathbb{C}}$. Thus the embedding $\varphi$ maps a loop $f$ into $\Omega\left(\mathbb{C}^{*}, \mathfrak{g}_{\mathbb{R}}\right) \times G_{\mathbb{R}}$, which are both tame Fréchet spaces. Now similar arguments apply.

Proposition 4.35. The group $M G_{\mathbb{C}} / M G_{\mathbb{R}}$ is a tame Fréchet manifold.

Proof. Review the embedding

$$
\psi: M G_{\mathbb{C}} \longrightarrow \Omega^{1}\left(\mathbb{C}^{*}, \mathfrak{g}_{\mathbb{C}}\right) \times G_{\mathbb{C}}
$$

A loop $f \cdot g$ is mapped onto $\psi(f \cdot g)=\left(g^{-1} f^{-1} d f g+g^{-1} d g,[f \cdot g](1)\right)$. This is the well-known gauge-action of the group $M G_{\mathbb{R}}$, denoted by $\mathcal{G}^{*}\left(M G_{\mathbb{R}}\right)$. Thus there is a well defined embedding

$$
\psi: M G_{\mathbb{C}} / M G_{\mathbb{R}} \longrightarrow \Omega^{1}\left(\mathbb{C}^{*}, \mathfrak{g}_{\mathbb{C}}\right) / \mathcal{G}^{*}\left(M G_{\mathbb{R}}\right) \times G_{\mathbb{C}} / G_{\mathbb{R}}
$$

No we study again the projections $\pi_{1}$ and $\pi_{2}$ on the first and second factor. $\pi_{2}$ is surjective; $G_{\mathbb{C}} / G_{\mathbb{R}}$ is a tame manifold; so this factor is no problem.

The projection on the first factor, $\pi_{1}$, needs a more careful analysis: the right multiplication of $M G_{\mathbb{R}}$ on $M G_{\mathbb{R}}$ is surjective: using the decomposition $\mathfrak{g}_{\mathbb{C}}=\mathfrak{g}_{\mathbb{R}}+i \mathfrak{g}_{\mathbb{R}}$, we get for $\Omega^{1}\left(\mathbb{C}^{*}, \mathfrak{g}_{\mathbb{C}}\right):=\Omega_{\mathbb{R}}^{1}\left(\mathbb{C}^{*}, \mathfrak{g}_{\mathbb{C}}\right)+i \Omega_{\mathbb{R}}^{1}\left(\mathbb{C}^{*}, \mathfrak{g}_{\mathbb{C}}\right)$.

The surjectivity of the right multiplication of $M G_{\mathbb{R}}$ on $M G_{\mathbb{R}}$ translates into the surjectivity of the $M G$-gauge action on the imaginary part $i \Omega_{\mathbb{R}}^{1}\left(\mathbb{C}^{*}, \mathfrak{g}_{\mathbb{C}}\right) \cap \Im\left(\pi_{1} \circ\right.$ $\psi)(M G)$. Thus we can suppose to have chosen a representative $f \in f \cdot M G$, such that the imaginary part $\pi_{1} \circ \psi(f)$ is 0 . So all we have to check is the real part. Here we find that $\exp ^{-1}(e)=0$. Thus $a_{-1}=0$. So we can identify the image $\pi_{1} \circ \psi\left(M G_{\mathbb{C}} / M G_{\mathbb{R}}\right) \simeq \Omega_{\mathbb{R}}^{1}\left(\mathbb{C}^{*}, \mathfrak{g} \mid a_{-1}=0\right)$. This is a tame Fréchet space.

Hence proposition 4.35 is proven. $\square$

Having proved that $M G_{\mathbb{C}}$ and $M G_{\mathbb{C}} / M G_{\mathbb{R}}$ are tame Fréchet manifolds we have to check that the same is true for the quotients $M G_{\mathbb{R}} / \operatorname{Fix}(\rho)$ and $M G_{D} / \operatorname{Fix}(\rho)$.

To this end, let $M G$ be a loop group and $M \rho$ the loop part of an involution of the second kind.

Proposition 4.36. Let $M G_{D}$ be a non-compact real form of $M G_{\mathbb{C}} . M G_{D}$ is a tame Fréchet manifold.

Proof. The group $M G_{D}$ is a closed subgroup of $M G_{\mathbb{C}}$ as it is the fixed point group of complex conjugation, hence some conjugate-linear involution $\square$

Proposition 4.37. The quotient spaces $M G_{\mathbb{R}} / F i x(M \rho)$ and $M G_{D, \rho} / F i x(M \rho)$ are tame manifolds. 
Proof. The proof is an argument analogous to the proof that $M G_{\mathbb{C}} / M G_{\mathbb{R}}$ is a tame Fréchet space.

The next class are twisted loop groups:

Proposition 4.38 (Twisted loop groups). Let $G$ be a compact simple Lie group of type $A_{n}, D_{n}$ or $E_{6}$ and $\sigma$ a diagram automorphism of order $m \in\{2,3\}$. Let $\omega=e^{\frac{2 \pi i}{m}}$.

- The group $A_{n} G^{\sigma}:=\left\{f \in A^{n} G \mid \sigma \circ f(z)=f(\omega z)\right\}$ is a Banach-Lie group.

- The group $M G^{\sigma}:=\{f \in M G \mid \sigma \circ f(z)=f(\omega z)\}$ is a tame manifold. Charts can be taken to be in $\Omega^{1}\left(\mathbb{C}^{*}, G\right)^{\sigma}$. Furthermore $M \mathfrak{g}^{\sigma} \simeq T_{e}\left(M G^{\sigma}\right)$.

Proof. To generalize the proofs of the non-twisted setting to the twisted setting one has to check that the subspaces defined by diagram automorphism are preserved by the logarithmic derivative.

1. For the exponential map, we use $\sigma_{\mathfrak{g}}$ resp. $\sigma_{G}$ to denote the realization of the diagram automorphism $\sigma$ on $\mathfrak{g}$ resp. $G$. Any involution of a semisimple Lie group satisfies the identity: $\sigma_{G} \circ \exp =\exp \circ \sigma_{\mathfrak{g}}$

$$
\left[\sigma_{G} \circ \operatorname{Mexp}(f)\right](z)=\exp \left(\sigma_{\mathfrak{g}}(f(z))\right)=\exp (f(\omega z))=[\operatorname{Mexp}(f)](\omega z),
$$

2. For the logarithmic derivative we calculate:

$$
\delta(\sigma \circ f)=(\sigma f)^{-1} d(\sigma \circ f)=\sigma f^{-1} \sigma d f=\sigma(\delta f) .
$$

Thus we get charts in the $\sigma$-invariant subalgebra of $\Omega^{1}\left(\mathbb{C}^{*}, \mathfrak{g}_{\mathbb{C}}\right)$.

The following definition is due to Omori [Omo97]:

Definition 4.39 (Exponential pair). A pair $(G, \mathfrak{g})$ consisting of a Fréchet group $G$ and a Fréchet space $\mathfrak{g}$ is called a topological group of exponential type if there is a continuous mapping:

$$
\exp : \mathfrak{g} \longrightarrow G
$$

Such that:

1. For every $X \in \mathfrak{g}, \exp (s X)$ is a one-parameter subgroup of $G$.

2. For $X, Y \in \mathfrak{g}, X=Y$ iff $\exp (s X)=\exp (s Y)$ for every $s \in \mathbb{R}$.

3. For a sequence $\left\{X_{n}\right\} \in \mathfrak{g}, \lim _{n \rightarrow \infty} X_{n}$ converges to an element $X \in \mathfrak{g}$ iff $\lim _{n \rightarrow \infty}\left(\exp s X_{n}\right)$ converges uniformly on each compact interval to the element $\exp (s X)$.

4. There is a continuous mapping Ad $: G \times \mathfrak{g} \longrightarrow \mathfrak{g}$ with $h \exp (s X) h^{-1}=$ $\exp s \operatorname{Ad}(h) X$ for every $h \in G$ and $X \in \mathfrak{g}$.

Proposition 4.40 (Exponential type). The pair $\left(M G_{\mathbb{K}}, M \mathfrak{g}_{\mathbb{K}}\right)$ is of exponential type.

Proof. We proved that $M G$ is a tame Fréchet Lie group, thus a topological group. To prove that $\left(M G_{\mathbb{K}}, M \mathfrak{g}_{\mathbb{K}}\right)$ is of exponential type, we have to check the four conditions given in definition 4.39:

1. The first condition can be checked by a pointwise analysis: for $f \in M \mathfrak{g}$ and for every $z \in \mathbb{C}^{*}$, the curve $\exp s f(z)$ is a 1-parameter subgroup in $G$. This pieces together for all $z \in \mathbb{C}^{*}$, to yield the condition. 
2. The second condition follows analogously: let $X, Y \in M \mathfrak{g} . \quad X=Y$ iff $X(z)=Y(z)$ for all $z \in \mathbb{C}^{*}$. The finite dimensional theory tells us that this is equivalent to the curves $\exp (s X(z)) \subset G_{\mathbb{K}}$ and $\exp (s Y(z)) \subset G_{\mathbb{K}}$ to be equivalent for all $z \in \mathbb{C}^{*}$, but this is equivalent to $\exp (s X)=\exp (s Y)$.

3. Let $\left\{X_{n}(z)\right\} \in M \mathfrak{g}, z \in \mathbb{C}$ be a sequence of elements such that $\lim _{n \rightarrow \infty} X_{n}(z)=$ $X \in M \mathfrak{g}$. Let $T \subset \mathbb{R}$ be a compact interval, $s \in T$. As we have on $M G$ the compact-open topology,

$$
\lim _{n \rightarrow \infty}\left(\exp s X_{n}\right)=\exp (s X) \Leftrightarrow \forall K \subset \mathbb{C}^{*}: \exp \lim _{n \rightarrow \infty}\left(\exp s X_{n}(K)\right)=\exp (s X)(K) .
$$

This assertion is correct as for every $z \in K: \exp \lim _{n \rightarrow \infty}\left(\exp s X_{n}(z)\right)=$ $\exp (s X)(z)$.

4. The last assertion follows again from pointwise consideration and the validity of the assertion for finite semisimple Lie groups.

We give some remarks about 1-parameter subgroups.

REMARK 4.41. Let $g(t):=X \exp (t u)$ for $u \in X \mathfrak{g}_{\mathbb{K}}$ be a 1-parameter subgroup in $X G_{\mathbb{K}}, X \in\left\{A_{n}, \mathbb{C}^{*}\right\}, \mathbb{K} \in\{\mathbb{R}, \mathbb{C}\}$. Then the following statements hold:

1. $X \exp (t u)_{z_{0}}$ is a 1-parameter group in $G_{\mathbb{C}}$ for all $z_{0} \in X$.

2. If $A_{n} \subset A_{n+k}$ then the embedding $A_{n+k} G \hookrightarrow A_{n} G$ maps 1-parameter subgroups onto 1-parameter subgroups.

Proof. Direct calculation.

REMARK 4.42. As we have seen, the fact that the exponential function does not define a local diffeomorphism is responsible for several difficulties; so it is reasonable to try to use a setting in which the exponential function defines a local diffeomorphism. So let us try to take loops $f: S^{1} \longrightarrow G$ satisfying some regularity condition. In this case the exponential map defines always a local diffeomorphism, as a neighborhood of the identity element of such a loop group is given by loops whose images lie in a small neighborhood $V$ of the identity of the subjacent Lie group; this neighborhood can be chosen in a way such that the group exponential is a diffeomorphism from an open neighborhood $U$ in the Lie algebra onto it. But now other problems appear:

1. Suppose the functions to be $H^{1}$-Sobolev loops. In this setting, one can construct weak Hilbert symmetric spaces of compact and non-compact type. Nevertheless, one cannot define the double extension corresponding to the $c$ and $d$-part of the Kac-Moody algebra. As this extension is responsible for the structure theory, this setting is not useful for us.

2. To be able to construct the extension corresponding to the derivative $d$, one needs loops that are $C^{\infty}$. For $C^{\infty}$-loops, it is possible to construct compact type symmetric spaces corresponding to the finite dimensional types $I$ and $I I I$, but for Kac-Moody symmetric spaces of $C^{\infty}$-loops there is no dualization: as the complexification of $c$ is not defined, we cannot complexify. As a consequence there are no symmetric spaces of the non-compact type.

The details of both theories are developed in [Pop05]. Summarizing we have the following observation:

Proposition 4.43. The setting of holomorphic loops is the biggest setting such that Kac-Moody symmetric space of the compact type and of the non-compact type of the same regularity condition can be defined. 
Let us mention in this context the short summary in [Ber03] about infinite dimensional differential geometry, where the conflict between often desirable structure as a Hilbert space and useful metrics is addressed.

5. Polar actions on tame Fréchet spaces. In this section we study polar representations of tame Fréchet affine loop groups on tame Fréchet vector spaces. Our aim is, to establish that the adjoint representation of Kac-Moody groups of holomorphic maps $\widehat{X G}$ and their associated $s$-representations induce polar actions on certain subspaces of $X \mathfrak{g}$; these subspaces are constructed as intersections of certain horospheres with spheres of constant norm; from the point of view of Kac-Moody geometry, our results assure, that there is a well-defined connection between the local linearized classes of objects such as polar actions and their principal orbits (that is isoparametric submanifolds) on the one hand and the global nonlinear classes of objects such as affine Kac-Moody symmetric spaces and twin cities on the other hand.

Let us sketch in a few lines the finite dimensional blueprint: Let $G$ be a simple compact Lie group and $K \subset G$ the fixed point group of some involution. Then the quotient space $M=G / K$ is a finite dimensional Riemannian symmetric space. The group $K$, called the isotropy group of $M$, stabilizes the point $p_{0} \equiv e K \in M$. Let $p \in M$. The linear representation $K \equiv K_{p}: T_{p} M \longrightarrow T_{p} M$ is called the isotropy representation of $M$. Recall furthermore that a representation $K: V \longrightarrow V$ of a Lie group $K$ on a vector space $V$ is polar, if there is a subspace $\Sigma \subset V$, called a section, that intersects each orbit orthogonally. It is an easy calculation, to check that the isotropy representation of a (finite dimensional) Riemann symmetric space is a polar representation of the isotropy group on the tangential space. Conversely, a theorem of J. Dadok states, that any polar representation is orbit equivalent to the isotropy representation of some symmetric space [Dad85]. For the isotropy representation of a symmetric space, possible choices of sections correspond to tangential spaces of maximal flats, that is maximal subspaces $F \subset M$, that are isometric to flat space. We show in forthcoming work, that affine Kac-Moody symmetric spaces behave in a similar way. Remark nevertheless a striking difference between the finite dimensional (spherical) and the infinite dimensional affine situation: while in the finite dimensional situation all orbits have finite codimensions, ranging from 2 to the rank, in the affine case there are orbits with (small) finite codimension bounded by $r k(G)$ and orbits with infinite codimension. This phenomenon is related to the dichotomy of complex affine Kac-Moody groups, that can be viewed either as (finite dimensional) (algebraic) groups over the ring of holomorphic functions or as (infinite dimensional) affine KacMoody group over the base field $\mathbb{C}$ (resp. $\mathbb{R}$. Via duality constructions this dichotomy is also the algebraic structure behind the differences in the geometry of coadjoint orbits of Kac-Moody groups and loop groups, which is sketched for example in the book [KW09]; we gave a building theoretic interpretation in [Fre12b], relating the finite dimensional orbits to the affine twin city and the infinite dimensional ones to the spherical building at infinity [Fre13d].

We prove in this section, that the orbits with finite codimension correspond to the gauge actions of tame loop groups on tame spaces; these actions are restrictions of the adjoint action of the Kac-Moody group to certain subspaces.

Closely related is the theory of polar actions on Hilbert spaces, which is described in the article [HPTT95, PT90, Ter95, Gro00].

The fundamental theorem due to C.-L. Terng states:

Theorem 5.1. Define $P(G, H):=\left\{g \in H^{1}([0,1], G) \mid(g(0), g(1)) \in H \subset G \times G\right\}$ 
and $V=H^{0}([0,1], \mathfrak{g})$. Suppose the $H$-action on $G$ is polar with flat sections. Let $A$ be a torus section through $e$ and let $\mathfrak{a}$ denote its Lie algebra. Then the gauge action of $P(G, H)$ on $V$ is polar with section $\mathfrak{a}$.

Proof. see [Ter95].

Important special cases are the following: let $\Delta_{\sigma} \subset G \times G$ denote the $\sigma$-twisted diagonal subgroup of $G \times G$, that is: $(g, h) \in \Delta_{\sigma}$ iff $h=\sigma(g)$. We use the notation $\Delta=\Delta_{\text {Id }}$ for the non-twisted subgroup.

1. The gauge action of $H^{1}$-Sobolev loop groups $P(G, G \times G) \cong H^{1}([0,1], G)$ on their $H^{0}$-Sobolev loop algebras $H^{0}([0,1], \mathfrak{g})$ is transitive.

2. The gauge action of $P\left(G, \Delta_{\sigma}\right)$ on $H^{1}([0,1], G)$ is polar with flat sections [HPTT95].

3. The gauge action of $P(G, K \times K)$ on $H^{1}([0,1], G)$ where $K$ is the fixed point set of some involution of $G$ is polar with flat sections [HPTT95].

4. The gauge action of $P\left(G, K_{1} \times K_{2}\right)$ on $H^{1}([0,1], G)$ where $K_{i}, i \in\{1,2\}$ are the fixed point groups of involutions of $G$ is polar with flat sections [HPTT95].

5. The gauge action of Sobolev- $H^{1}$-loop groups $H^{1}\left(S^{1}, G\right)$ on their Sobolev $H^{0}$ loop algebras $H^{0}\left(S^{1}, \mathfrak{g}\right)$-is polar [PT88].

In this section we describe a similar theory for the loop groups $X G^{\sigma}$ on the tame loop algebras $X \mathfrak{g}^{\sigma}$. As usual let $X \in\left\{A_{n}, \mathbb{C}^{*}\right\}$. Recall, that holomorphic functions on $A_{n}$ are assumed to be holomorphic in an open set containing $A_{n}$. From the embedding $X G^{\sigma} \hookrightarrow H^{1}([0,1], G)$ and $X \mathfrak{g}^{\sigma} \hookrightarrow H^{0}([0,1], \mathfrak{g})$ it is clear that the algebraic part of the theory is independent from the actual regularity conditions imposed. This means for example: sections for a polar action on the subspaces of holomorphic functions $X \mathfrak{g}$ correspond to sections for the polar actions on some Hilbert space of $H^{0}$-functions $L^{0} \mathfrak{g}$; consequently also further objects, defined from the polar actions such as the associated affine Weyl groups are the same. Hence the crucial point in establishing the existence of polar actions on some algebra $X \mathfrak{g}$ is, to check that the additional regularity restrictions fit, hence most importantly, that each orbits intersects a fixed section. This breaks down to two points:

1. One has to show locally that the additional regularity conditions are satisfied.

2. One has to show globally that monodromy conditions are satisfied.

Leaving the realm of Hilbert spaces, a remark to the definition of orthogonality is necessary: While there are still scalar products on Fréchet spaces, the space is no longer complete with respect to the metric defined by the scalar product. Hence to define orthogonality on $X \mathfrak{g}$ we use the $H^{0}$-scalar product induced on $M \mathfrak{g}$ by the embedding into $H^{0}([0,1], \mathfrak{g})$. Tautologically, the embedding $X \mathfrak{g} \hookrightarrow L^{0} \mathfrak{g}$ is an embedding. In consequence we can define polar actions on Banach (resp. tame) spaces like that:

Definition 5.2. An action of a Lie group $G$ on a Fréchet space $F$ is called polar iff there is a subspace $S$, called a section, intersecting each orbit orthogonally with respect to some scalar product.

TheOREm 5.3. The gauge action of $X G_{\mathbb{R}}^{\sigma}$ on $X \mathfrak{g}^{\sigma}$ is polar; an Abelian subalgebra $\mathfrak{a} \subset \mathfrak{g}$ interpreted as constant loops is a section.

The proof consists of two parts:

1. We have to show that each orbit intersects the section $\mathfrak{a}$.

2. We have to show that the intersection is orthogonal.

The second part follows trivially from the embedding and Terng's result. 
Thus we are left with proving the first assertion. We do this in a step-by-step way: first we study the action of $C^{k}$-loop groups on $C^{k-1}$-loop algebras $(k \in\{\mathbb{N}, \infty\})$. Then we proceed to the holomorphic setting of theorem 5.3.

Lemma 5.4. The gauge action of $L^{k} G^{\sigma}$ on $L^{k-1} \mathfrak{g}^{\sigma}$ is polar for $k \in\{\mathbb{N}, \infty\}$.

This result is used without proof in [Pop05] in order to show that all finite dimensional flats are conjugate. We do not know if a proof can be found in the literature. For completeness we give one:

Proof of lemma 5.4 .

1. Orthogonality in $L^{k-1} \mathfrak{g}^{\sigma}$ is defined via the embedding into the space $H^{0}([0,1], \mathfrak{g})$ and the use of the $H^{0}$-scalar product. Hence orthogonality of the intersection between sections and orbits is covered by Terng's result.

\section{Local regularity}

Define the following spaces

$$
P(G, H)^{k}:=\left\{g \in C^{k}([0,1], G) \mid(g(0), g(1)) \in H \subset G \times G\right\} .
$$

Furthermore we use the equivalence [Ter95]

$$
\begin{aligned}
P(G ; e \times G) & \simeq H^{0}([0,1], \mathfrak{g}), \\
h & \leftrightarrow-h^{\prime} h^{-1} .
\end{aligned}
$$

Terng's polarity result [Ter95] yields that the action of $P\left(G, \Delta_{\sigma}\right)$ on $P(G ; e \times$ $G$ ) defined by $(g(t), h(t)) \mapsto g(t) h(t) g(0)^{-1}$ is polar with a section of constant loops exp $t \mathfrak{a}$ where $\mathfrak{a}$ is a maximal Abelian subalgebra in $\mathfrak{g}$ (if $\sigma \neq 0$ we restrict to $\mathfrak{a}_{\sigma}$ and omit the $\sigma$ in the notation [Kac90]). Thus for every $h(t) \in P(G ; e \times$ $G)$ there exist $g(t) \in P\left(G, \Delta_{\sigma}\right)$ and $X \in \mathfrak{a}$, such that $g(t) h(t) g(0)^{-1}=$ $\exp (t X)$.

Rearranging this equation we deduce for any loop $g(t) \in P\left(G, \Delta_{\sigma}\right)$ the explicit description $g(t):=\exp (t X) g(0) h(t)^{-1}$. Hence if $h(t) \in P^{k}(G ; e \times G)$ then $g(t) \in P^{k}\left(G, \Delta_{\sigma}\right)$. Combining this with the orthogonality we obtain that the actions of $P^{k}\left(G, \Delta_{\sigma}\right)$ on $P^{k}(G ; e \times G) \simeq H^{k-1}([0,1], \mathfrak{g})$ and of $P^{\infty}\left(G, \Delta_{\sigma}\right)$ on $P^{\infty}(G ; e \times G) \simeq H^{\infty}([0,1], \mathfrak{g})$ are polar.

3. The periodicity relation: We want to show that $L^{k} G^{\sigma}$ acts on $L^{k-1} \mathfrak{g}^{\sigma}$ with slice $\mathfrak{a}$ for $k \in\{\mathbb{N}, \infty\}$.

(a) Let first $g \in L^{k} G^{\sigma}$ and $u \in L^{k-1} \mathfrak{g}^{\sigma}$. Then $g \cdot u=g u g^{-1}-g^{\prime} g^{-1}$ is in $L^{k-1} \mathfrak{g}^{\sigma}$. Thus $L^{k} G^{\sigma}$ acts on $L^{k-1} \mathfrak{g}^{\sigma}$.

(b) We have to show that any $L^{k} G^{\sigma}$-orbit intersects the section $\mathfrak{a}$. This is equivalent to: For each $u \in L^{k-1} \mathfrak{g}^{\sigma}$, there is $X \in \mathfrak{g}$ and $g \in P^{k}(G, \Delta)$ such that $\exp (t X)=g(t) h(t) g^{-1}(0)$ with $h^{\prime}(t)=u(t) h(t)$ and the derivatives coincide; interpret in this last equation $u(t)$ as a quasi-periodic function on $\mathbb{R}$ (i.e. $u(t+2 \pi)=\sigma u(t)$ and $h(t)$ as a function on $\mathbb{R}$ ).

Using the first part, we find a function $g(t) \in P^{k}\left(G, \Delta_{\sigma}\right)$. Hence, what remains is to check the closing condition of the derivatives: $g^{(n)} \cdot u(2 \pi)=$ $\sigma g^{(n)} \cdot u(0)$. We prove that it is equivalent to the closing condition $g^{(n+1)}(2 \pi)=\sigma g^{(n+1)}(0)$.

We start with the case $n=1$. For this case, we have to show

$$
\exp ((t+2 \pi) X) g_{0} h(t+2 \pi)^{-1}=\sigma\left(\exp (t X) g_{0} h(t)^{-1}\right) .
$$


After rearranging, this is equivalent to the identity

$$
\sigma\left(g_{0}^{-1}\right) \exp (2 \pi X) g_{0}=\sigma\left(h(t)^{-1}\right) h(t+2 \pi) .
$$

As the left side is a constant we find:

$$
\left(\sigma\left(h(t)^{-1}\right) h(t+2 \pi)\right)^{\prime}=0 .
$$

Hence: $-\sigma\left(h(t)^{-1} h^{\prime}(t) h(t)^{-1}\right) h(t+2 \pi)+\sigma\left(h(t)^{-1}\right) h^{\prime}(t+2 \pi)=0$. Rearranging this equality we get

$$
\sigma(u(t))=-\sigma\left(h^{\prime}(t)\right) \sigma\left(h(t)^{-1}\right)=-h^{\prime}(t+2 \pi) h(t+2 \pi)^{-1}=u(t+2 \pi)
$$

which is the desired periodicity condition.

For $n \neq 0$ we use induction. If $g$ is $k$-times differentiable then $u$ is $k-1$-times differentiable. This proves the lemma.

Proof of theorem 5.3. To prove the theorem, we have to further strengthen the used regularity conditions to holomorphic functions. The description in the proof of lemma 5.4 shows that the group of analytic loops $L_{a n}\left(S^{1}, G\right)$ acts polarly with section $\mathfrak{a}$ on the algebra $L_{a n}\left(S^{1}, \mathfrak{g}\right)$ of analytic loops.

1. The case of holomorphic loops on $\mathbb{C}^{*}$ For the specialization to holomorphic maps we use the description:

$$
H_{\mathbb{C}^{*}}\left(\mathbb{C}, \mathfrak{g}_{\mathbb{C}}\right)_{\mathbb{R}}:=\left\{f: \mathbb{C} \longrightarrow \mathfrak{g}_{\mathbb{C}} \mid f(z+i \mathbb{Z})=f(z), f i \mathbb{R} \subset \mathfrak{g}_{\mathbb{R}}\right\} .
$$

Identifying it $\leftrightarrow t$ in this (resp. the above) description we get an embedding:

$$
H_{\mathbb{C}^{*}}\left(\mathbb{C}, \mathfrak{g}_{\mathbb{C}}\right)_{\mathbb{R}} \hookrightarrow H^{\infty}\left(S^{1}, \mathfrak{g}\right)
$$

This shows that there are no problems concerning the monodromy. So we have only to check the regularity aspect. For $g \in M G$ and $u \in M \mathfrak{g}, g$. $(u)=g u g^{-1}-g^{\prime} g^{-1} \in M \mathfrak{u}$. On the other hand, using the description in the proof of lemma 5.4, we get for $u \in M \mathfrak{g}$ a transformation function $g(t):=$ $\exp (t X) g(0) h(t)^{-1}$. A priory this function is in $L\left(S^{1}, G\right)$; but $\exp (t X)$ can be continued to a holomorphic function on $\mathbb{C}, g(0)$ is a constant and $h(t)^{-1}$ is a solution of the differential equation: $h^{\prime}(t)=u(t) h(t)$; if $u(t)$ is defined on $\mathbb{C}^{*}$, this equation has a solution on the universal cover of $\mathbb{C}^{*}$, that is $\mathbb{C}$. So $g(t)$ is defined on $\mathbb{C}$, but has perhaps nontrivial monodromy; this is, of course, not possible, as the embedding tells us that $g(t) \subset L_{a n} G$.

2. The case of holomorphic loops on $A_{n}$ This case is exactly similar. $\mathbb{C}$ is replaced by $A^{\prime}$ (compare subsection 3.1).

Hence theorem 5.3 is proved.

Thus we have proven that $\sigma$-actions and diagonal actions are polar. Those two cases corresponds to the isotropy representation of Kac-Moody symmetric spaces of types $I I$ and $I V$ : the diagonal action is induced by the isotropy representation of Kac-Moody symmetric spaces in the non-twisted case, the $\sigma$-action is induced by the isotropy representation of $\sigma$-twisted one.

The isotropy representations of Kac-Moody symmetric spaces of type $I$ and $I I I$ correspond to the Hermann examples. A holomorphic version of the Hermann examples [HPTT95] can be defined in exactly the same way: 
Let $X G_{\mathbb{R}}^{\sigma}$ be a simply connected loop group, $\rho$ an involution such that $X \mathfrak{g}_{\mathbb{R}}^{\sigma}=$ $\mathcal{K} \oplus \mathcal{P}$ is the decomposition into the \pm 1 -eigenspaces of the involution induced by $\rho$ on $X \mathfrak{g}_{\mathbb{R}}^{\sigma}$. Let $X K_{\mathbb{R}} \subset X G_{\mathbb{R}}^{\sigma}$ be the subgroup fixed by $\rho$.

THEOREM 5.5. The gauge action of $X K_{\mathbb{R}}$ on $\mathcal{P}$ is polar.

Proof. The proof is like the one of theorem 5.3. One starts with a similar result for polar actions on Hilbert action [Ter95] and checks then step by step that the introduced higher regularity conditions fit together.

6. Tame structures on Kac-Moody algebras. In this section we describe explicit realizations as central extensions of holomorphic loop algebras of the abstract affine geometric Kac-Moody algebras which we introduced in definition 4.1.

Let $X \in\left\{A_{n}, \mathbb{C}^{*}\right\}$. As usual, holomorphic functions on $A_{n}$ are understood to be holomorphic in an open set containing $A_{n}$.

Definition 6.1 (holomorphic affine geometric Kac-Moody algebra). Define $\widehat{X \mathfrak{g}}$ to be an explicit realization of $\widehat{L}(\mathfrak{g}, \sigma)$ with $L(\mathfrak{g}, \sigma)$ in the category of holomorphic maps $X \mathfrak{g}^{\sigma}$.

Thus an element of a Kac-Moody algebra can be represented by a triple

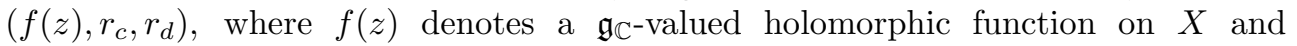
$\left\{r_{c}, r_{d}\right\} \in \mathbb{C}$.

We equip those algebras with the norms $\|\widehat{f}\|_{n}=\left\|\left(f, r_{c}, r_{d}\right)\right\|_{n}:=\sup _{z \in A_{n}}\left|f_{z}\right|+$ $\left(r_{c} \bar{r}_{c}+r_{d} \bar{r}_{d}\right)^{\frac{1}{2}}$. Thus we use the supremum norm on the loop algebra and complete it with an Euclidean norm on the double extension defined by $c$ and $d$.

Lemma 6.2 (Banach- and Fréchet structures on Kac-Moody algebras).

1. For each $n$, the algebras $\widehat{A_{n} \mathfrak{g}_{\mathbb{R}}}$ and $\widehat{A_{n}} \mathfrak{g}_{\mathbb{C}}$ equipped with the norm \|\|$_{n}$ are Banach-Lie algebras,

2. $\widehat{M g}_{\mathbb{R}}^{\sigma}$ and $\widehat{M g}_{\mathbb{C}}^{\sigma}$ equipped with the sequence of norms \|\|$_{n}$ are tame Fréchet-Lie algebras.

Proof. Let $\mathbb{F} \in\{\mathbb{R}, \mathbb{C}\}$.

1. As a consequence of lemma $4.5, A_{n} \mathfrak{g}^{\sigma}$ is a Banach space. Thus $\widehat{A n n}^{\sigma}$ is Banach.

To prove that $a d\left(f+r_{c} c+r_{d} d\right)$ is continuous, we use $\left[f+r_{c} c+r_{d} d, g+s_{c} c+\right.$ $\left.s_{d} d\right]=[f, g]+r_{d}[d, g]-s_{d}[d, f]=[f, g]_{0}+\omega(f, g) c+r_{d} i z g^{\prime}-s_{d} i z f^{\prime}$. Continuity follows from the continuity of $\frac{d}{d z}$, which is a consequence of the Cauchyinequality and the boundedness of multiplication on compact domains.

2. $M \mathfrak{g}^{\sigma}$ is a tame Fréchet space as a consequence of lemma 4.5. Thus $\widehat{M \mathfrak{g}}^{\sigma}$ is tame as a direct product of tame spaces (lemma 2.15). To prove tameness of the adjoint action, we need tame estimates for the norms. Those estimates follow directly from the Banach space situation:

$$
\begin{aligned}
& \left\|a d\left(f+r_{c} c+r_{d} d\right)\left(g+s_{c} c+s_{d} d\right)\right\|_{n}= \\
& =\left\|[f, g]_{0}+\omega(f, g) c+r_{d} i z g^{\prime}-s_{d} i z f^{\prime}\right\|_{n} \leq \\
& \leq 2\|f\|_{n}\|g\|_{n}+\|\omega(f, g) c\|_{n}+\left\|r_{d} z g^{\prime}\right\|_{n}+\left\|s_{d} z f^{\prime}\right\|_{n} \leq \\
& \leq 2\|f\|_{n}\|g\|_{n}+\|f\|_{n}\left\|g^{\prime}\right\|_{n}+\left|r_{d}\right|\|z\|_{n}\left\|g^{\prime}\right\|_{n}+\left|s_{d}\right|\|z\|_{n}\left\|f^{\prime}\right\|_{n} \leq \\
& \leq 2\|f\|_{n}\|g\|_{n}+\|f\|_{n} \frac{e^{n+1}}{e-1}\|g\|_{n+1}+\left|r_{d}\right| e^{n} \frac{e^{n+1}}{e-1}\|g\|_{n+1}+\left|s_{d}\right| e^{n} \frac{e^{n+1}}{e-1}\|f\|_{n+1} \leq \\
& \leq\left(2+\frac{e^{n+1}}{e-1}+2 \frac{e^{2 n+1}}{e-1}\right)\|\widehat{f}\|_{n+1}\|\widehat{g}\|_{n+1} \leq 5 e^{2 n+1}\|\widehat{f}\|_{n+1}\|\widehat{g}\|_{n+1} .
\end{aligned}
$$


Thus $\operatorname{ad}(\widehat{g})$ is $\left(1,0,5 e^{2 n+1}\|\widehat{f}\|_{n+1}\right)$-tame.

This result shows that the tame structure on the Kac-Moody algebra is preserved by the adjoint action. For additional analytic details and the Cauchy-inequalities see for example [BG91].

\section{Affine Kac-Moody groups.}

7.1. The loop group construction of affine Kac-Moody groups. In this section we describe the construction of affine Kac-Moody groups as 2-dimensional extensions of loop groups. Our presentation of the central extensions follows a construction proposed [PS86] for a Hilbert space setting; we use the tame Fréchet setting, developed in section 4. Furthermore using a technical result of B. Popescu we prove that Kac-Moody groups of holomorphic loops carry a structure as tame Fréchet manifolds.

Let $G_{\mathbb{C}}$ denote a complex semisimple Lie group and $G$ its compact real form. As the constructions are valid in Kac-Moody groups defined with respect to various different regularity conditions, we use the regularity-independent notation $L\left(G_{\mathbb{C}}, \sigma\right)$ for the complex loop group and $L(G, \sigma)$ for its real form of compact type. To define groups of polynomial or analytic loops, we use the fact that every compact Lie group is isomorphic to a subgroup of some unitary group. Hence we can identify it with a matrix group. Similarly the complexification can be identified with a subgroup of some general linear group [PS86]. Groups of polynomial loops are then defined with respect to this representation.

Kac-Moody groups are constructed in two steps.

1. The first step consists in the construction of an $S^{1}$-bundle in the real case (resp. a $\mathbb{C}^{*}$-bundle in the complex case) that corresponds via the exponential map to the central term $\mathbb{R} c$ (resp. $\mathbb{C} c$ ) of the Kac-Moody algebra.

2. In the second step we construct a semidirect product with $S^{1}$ (resp. $\left.\mathbb{C}^{*}\right)$. This corresponds via the exponential map to the $\mathbb{R} d$ - (resp. $\mathbb{C} d$-) term

Study first the extension of $L(G, \sigma)$ with the short exact sequence:

$$
1 \longrightarrow S^{1} \longrightarrow \widetilde{L}(G, \sigma) \longrightarrow L(G, \sigma) \longrightarrow 1 \text {. }
$$

There are various groups $X$ that fit into this sequence. We need to define $\widetilde{L}(G, \sigma)$ in a way that its tangential Lie algebra at the identity $e \in \widetilde{L}(G, \sigma)$ is isomorphic to $\widetilde{L}(\mathfrak{g}, \sigma)$.

As described in [PS86] this $S^{1}$-bundle is best represented by triples: take triples $(g(z), p(z, t), w)$ where $g(z)$ is an element in the loop group, $p(z, t)$ a path connecting the identity to $g(z)$ and $w \in S^{1}$ (respective $w \in \mathbb{C}^{*}$ ) subject to the relation of equivalence: $\left(g_{1}(z), p_{1}(z, t), w_{1}\right) \sim\left(g_{2}(z), p_{2}(z, t), w_{2}\right)$ iff $g_{1}(z)=g_{2}(z)$ and $w_{1}=$ $C_{\omega}\left(p_{2} * p_{1}^{-1}\right) w_{2}$. The term $w_{1}=C_{\omega}\left(p_{2} * p_{1}^{-1}\right) w_{2}$ defines a twist of the bundle. Here we put:

$$
C_{\omega}\left(p_{2} * p_{1}^{-1}\right)=e^{\int_{S\left(p_{2} * p_{1}^{-1}\right)} \omega}
$$

where $S\left(p_{2} * p_{1}^{-1}\right)$ is a surface bounded by the closed curve $p_{2} * p_{1}^{-1}$ and $\omega$ denotes the 2 -form used to define the central extension of $L(\mathfrak{g}, \sigma)$. Group multiplication is defined by

$\left(g_{1}(z), p_{1}(z, t), w_{1}\right) \cdot\left(g_{2}(z), p_{2}(z, t), w_{2}\right)=\left(g_{1}(z) g_{2}(z), p_{1}(z, t) * g_{1}(z) \cdot p_{2}(z, t), w_{1} w_{2}\right)$. 
If $G$ is simply connected it can be shown that this object is a well defined group independent of arbitrary choices made in the construction iff $\omega$ is integral. This condition is satisfied by our definition of $\omega$ [PS86], theorem 4.4.1. If $G$ is not simply connected, the situation is a little more complicated: let $G=H / Z$ where $H$ is a simply connected Lie group and $Z=\pi_{1}(G)$. Let $(L G)_{0}$ denote the identity component of $L G$. We can describe the extension using the short exact sequence:

$$
1 \longrightarrow S^{1} \longrightarrow \widetilde{L H} / Z \longrightarrow(L G)_{0} \longrightarrow 1
$$

see [PS86], section 4.6. .

In case of complex loop groups, the $S^{1}$-bundle is replaced by a $\mathbb{C}^{*}$-bundle.

Hence, we can now give the definition of Kac-Moody groups:

DeFinition 7.1 (Kac-Moody group).

1. The real Kac-Moody group $\widehat{M G}_{\mathbb{R}}$ is the semidirect product of $S^{1}$ with the $S^{1}$-bundle $\widetilde{M G} \mathbb{R}$.

2. The complex Kac-Moody group $\widehat{M G}_{\mathbb{C}}$ is its complexification: a semidirect

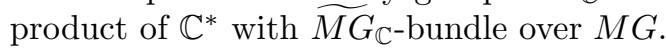

The action of the semidirect $S^{1}$ (resp. $\mathbb{C}^{*}$ )-factor is in both cases defined by a shift of the argument:

$$
\mathbb{C}^{*} \ni w_{d}: M G \rightarrow M G: f(z) \mapsto f\left(z w_{d}\right)
$$

REMARK 7.2. Remark that in the compact case the shift is by elements $w_{d}=e^{i \varphi_{d}}$ only. Hence the action preserves the unit circle $S^{1}$. Thus one can use function spaces on $S^{1}$ thus yielding more general Kac-Moody groups than the groups of holomorphic loops, we study. Nevertheless those groups have no complexification in the same regularity class.

The next aim is to prove that Kac-Moody groups are tame Fréchet manifolds. To this end we use a result of B. Popescu [Pop05] stating that fiber bundles whose fiber is a Banach space over tame Fréchet manifolds are tame.

We start with the definition of tame fiber bundles:

Definition 7.3 (tame Fréchet fiber bundle). A fiber bundle $P$ over $M$ with fiber $G$ is a tame Fréchet manifold $P$ together with a projection map $\pi: P \longrightarrow M$ satisfying the following condition:

For each point $x \in M$ there is a chart $\varphi: U \longrightarrow V \subset F$ with values in a tame Fréchet space $F$ such that there is a chart $\varphi: \pi^{-1}(U) \longrightarrow G \times U \subset G \times F$ such that the projection $\pi$ corresponds to a projection of $U \times F$ onto $U$ in each fiber.

The following lemma is proved in [Pop05].

Lemma 7.4. Let $P$ be a fiber bundle over $M$ whose fiber is a Banach manifold; then $P$ is a tame Fréchet manifold.

This result contains the important corollary:

COROLlary 7.5. The Kac-Moody groups $\widehat{M G}_{\mathbb{R}}, \widehat{M G}_{\mathbb{C}}$, the quotient spaces $\widehat{M G}_{\mathbb{C}} / \widehat{M G}_{\mathbb{R}}, \widehat{M G}_{D}$ and the quotient spaces $\widehat{M G}_{\mathbb{R}} /$ Fix $(\rho)$ and $\widehat{M G}_{D} /$ Fix $(\rho)$ are tame Fréchet manifolds.

Next we prove: 
THEOREM 7.6. $\widehat{M G}_{\mathbb{C}} / \widehat{M G}_{\mathbb{R}}$ is diffeomorphic to a vector space.

Proof. By theorem 4.35, we know that $M G_{\mathbb{C}} / M G_{\mathbb{R}}$ is diffeomorphic to a vector space. As $M G_{\mathbb{R}}$ is a subgroup of $M G_{\mathbb{C}}$, the quotient is well defined. To prove the theorem we check the decomposition

$$
\widehat{M G}_{\mathbb{C}} / \widehat{M G}_{\mathbb{R}} \simeq M G_{\mathbb{C}} / M G_{\mathbb{R}} \times\left(\mathbb{R}^{+}\right)^{2} .
$$

To this end we use the description of the elements in $\widehat{M G}_{\mathbb{C}} / \widehat{M G}$ as 4 -tuples. Two 4-tuples $\left(g(z), p(z, t), r_{c}, r_{d}\right)$ and $\left(g^{\prime}(z), p^{\prime}(z, t), r_{c}^{\prime}, r_{d}^{\prime}\right)$ describe the same element of $\widehat{M G}_{\mathbb{C}} / \widehat{M G}$ iff there exists an element $\left(h(z), q(z, t), s_{c}, s_{d}\right) \in M G_{\mathbb{R}}$ such that

$$
\left(g(z), p(z, t), r_{c}, r_{d}\right)=\left(g^{\prime}(z)+h(z), p^{\prime}(z, t)+q(z, t), r_{c}^{\prime}+s_{c}, r_{d}^{\prime}+s_{d}\right) .
$$

Hence the equivalence classes for $g(z)$ are elements of $M G_{\mathbb{C}} / M G_{\mathbb{R}}$. The extension of $M G_{\mathbb{R}}$ lies in $S^{1}$. Thus $r_{c}$ and $r_{d}$ are defined up to an element in $S^{1}$. So we obtain a description of $\hat{M G}_{\mathbb{C}} / \widehat{M G}_{\mathbb{R}}$ as a $\left(\mathbb{R}^{+}\right)^{2}$-bundle over $M G_{\mathbb{C}} / M G_{\mathbb{R}}$.

As $M G_{\mathbb{C}} / M G_{\mathbb{R}}$ is diffeomorphic to a vector space (and hence simply connected), this bundle is trivial. The diffeomorphism can be described by mapping $\left(g(z), p(z, t), r_{c}, r_{d}\right)$ onto $\left(g(z), r_{c}, r_{d}\right)$, hence by forgetting the path $p(z, t)$.

Now we investigate the quotients $\widehat{M G} / \widehat{\operatorname{Fix}(\sigma)}$.

The group $\widehat{\operatorname{Fix}(\sigma)}$ consists of elements $\left(g, p, r_{c}, r_{d}\right) \in \widehat{M G}$ such that $\{g, p \in$ $\left.\operatorname{Fix}(\sigma), r_{c} \in \pm 1, r_{d} \in \pm 1\right\}$. As $\left\{r_{c}, r_{d}\right\} \in \pm 1$, this is a covering with four leaves. The details of the argument follow the description found in [Pop05] for the smooth $C^{\infty}$-setting.

Let $H \subset M G_{\mathbb{C}}$ be a real form of non-compact type.

The description of the space $H / \operatorname{Fix}(\sigma) \subset M G_{\mathbb{C}} / \operatorname{Fix}(\sigma)$ follows similarly. This yields a proof of the following theorem:

THEOREM 7.7. The space H/Fix $(\sigma)$ is diffeomorphic to a vector space.

In section 4.3 we introduced the pointwise exponential function Mexp : $\widehat{M g} \longrightarrow$ $\widehat{M G}$. This exponential function can be extended to an exponential function on the Kac-Moody algebra $\widehat{M \mathfrak{g}}$.

$$
\widehat{\operatorname{Mexp}}: \widehat{M g} \longrightarrow \widehat{M G}
$$

Via $\widehat{\text { Mexp }}$ the central extension $\mathbb{C} c$ corresponds to the fiber of the $\mathbb{C}^{*}$-bundle and the $\mathbb{C} d$-term corresponds to the $\mathbb{C}^{*}$-factor of the semidirect product.

To study more precisely the properties of the exponential function, we introduce the notion of curves in a Kac-Moody group. As a Kac-Moody group $\widehat{M G}_{\mathbb{R}}$ is locally a (topologically) direct product of the loop group $M G_{\mathbb{R}}$ with $\mathbb{R}^{2}$, a path: $\widehat{\gamma}:(-\epsilon, \epsilon) \rightarrow$ $\widehat{M G}$ is locally described by three components: $\widehat{\gamma}(t)=\left(\gamma(t), \gamma_{c}(t), \gamma_{d}(t)\right)$, with $\gamma(t)$ taking values in $M G_{\mathbb{R}}$ and $\gamma_{c}(t), \gamma_{d}(t)$ taking values in $\mathbb{R}$. For every $z \in \mathbb{C}^{*}, \gamma$ defines a path $\gamma_{z}(t):(-\epsilon, \epsilon) \rightarrow G$ by setting $\gamma_{z}(t):=[\gamma(t)](z)$. A path $\gamma:(-\epsilon, \epsilon) \rightarrow M G$, $t \rightarrow \gamma(t)$ is differentiable (respective smooth) iff the map $\delta:(-\epsilon, \epsilon) \times \mathbb{C}^{*} \rightarrow G_{\mathbb{C}}$, $(t, z) \rightarrow \delta(t, z)$ such that $\delta(t, z)=\gamma_{z}(t)$ is differentiable (respective smooth).

For the group $\widetilde{M G}$ we can describe the exponential function as follows:

Proposition 7.8. The exponential function $\widetilde{\mathrm{Mexp}}: \widetilde{M \mathfrak{g}} \longrightarrow \widetilde{M G}$ is defined by

$$
\widehat{\operatorname{Mexp}}\left(f+r_{c} c\right)=\left(\operatorname{Mexp}(f),\left.(t \longrightarrow \operatorname{exptu})\right|_{0} ^{1}, r^{i r_{c}}\right) \text {. }
$$


For the straightforward proof one has to check that this defines a 1-parameter subgroup whose differential at the identity is $f+r_{c} c$.

7.2. The Adjoint action. Similarly to finite dimensional simple Lie groups a Kac-Moody group admits an adjoint action on its Lie algebra:

EXAmple 7.9 (Adjoint action). With $x=\{w,(g, p, z)\}$, the Adjoint action of $\widehat{M G}^{\sigma}$ on $\widehat{M}^{\sigma}$ is described by the following formulae

$$
\begin{aligned}
& \operatorname{Ad}(x) u:=g w(u) g^{-1}+\left\langle g w(u) g^{-1}, g^{\prime} g^{-1}\right\rangle c \\
& \operatorname{Ad}(x) c:=c \\
& \operatorname{Ad}(x) d:=d-g^{\prime} g^{-1}+\frac{1}{2}\left\langle g^{\prime} g^{-1}, g^{\prime} g^{-1}\right\rangle c .
\end{aligned}
$$

Here $\omega(u)$ denotes the shift of the argument by $\omega$.

For the proof compare [HPTT95], [PS86], and [Kac90].

Proof.

- $c$ generates the center, thus $\operatorname{Ad}(x) c:=c$.

- $\operatorname{Ad}(x) u$ follows by integrating the $A d$-action.

- To calculate $\operatorname{Ad}(g)(d)$, we use the $A d$-invariance of the Lie bracket. As $[d, v]=$ $v^{\prime}$ we get for all $v \in L_{a l g} \mathfrak{g}^{\sigma}$ :

$$
\begin{aligned}
& g v^{\prime} g^{-1}+\left\langle g v^{\prime} g^{-1}, g^{\prime} g^{-1}\right\rangle c= \\
= & \operatorname{Ad}(g)\left(v^{\prime}\right)=\operatorname{Ad}(g)[d, v]=[\operatorname{Ad}(g)(d), \operatorname{Ad}(g)(v)]= \\
= & {\left[h+\mu c+\nu d, g v g^{-1}+\left\langle g v g^{-1}, g^{\prime} g^{-1}\right\rangle c\right]=} \\
= & {\left[h, g v g^{-1}\right]+\nu\left[d, g v g^{-1}\right]=} \\
= & h g v g^{-1}-g v g^{-1} h+\omega\left(h,\left(g v g^{-1}\right)^{\prime}\right) c+\nu g^{\prime} v g^{-1}+\nu g v^{\prime} g^{-1}+\nu g v\left(g^{-1}\right)^{\prime},
\end{aligned}
$$

with $h \in L_{\text {alg }} \mathfrak{g}^{\sigma}$ and $\{\mu, \nu\} \in \mathbb{R}$.

To get equality we have to choose $\nu=1, h=-g^{\prime} g^{-1}$. This gives us

$$
\begin{aligned}
& h g v g^{-1}-g v g^{-1} h+\omega\left(h,\left(g v g^{-1}\right)^{\prime}\right) c+\nu g^{\prime} v g^{-1}+\nu g v^{\prime} g^{-1}+\nu g v\left(g^{-1}\right)^{\prime}= \\
= & -g^{\prime} v g^{-1}+g v g^{-1} g^{\prime} g^{-1}+\omega\left(-g^{\prime} g^{-1},\left(g v g^{-1}\right)^{\prime}\right) c+g^{\prime} v g^{-1}+g v^{\prime} g^{-1}+g v\left(g^{-1}\right)^{\prime}= \\
= & \omega\left(-g^{\prime} g^{-1},\left(g v g^{-1}\right)^{\prime}\right) c+g v^{\prime} g^{-1} .
\end{aligned}
$$

Thus we are left with the calculation of $\mu$. To this end we use the property that $A d$ acts by isometries. This results in $\mu=\frac{1}{2}\left\langle g^{\prime} g^{-1}, g^{\prime} g^{-1}\right\rangle$.

More details can be found in [PS86, HPTT95, Pop05, Pop06].

THEOREM 7.10 (Polarity of the Adjoint action). Let $H_{l, r} \subset \widehat{X \mathfrak{g}}^{\sigma},\{l, r\} \in$ $\mathbb{R} \times \mathbb{R} \backslash\{0\}$ denote the intersection of the sphere with radius $-l^{2}$ with the horosphere $r_{d}=r$. The restriction of the Adjoint action to $H_{l, r}$ is polar.

Proof. The restriction of the Adjoint action to $H_{l, r}$ coincides with the gauge action on $X \mathfrak{g}$. Hence, theorem 7.10 is a direct consequence of theorem 5.3.

C.-L. Terng describes how to associate an affine Weyl group to this gauge action [Ter95]. This is exactly the affine Weyl group of the Kac-Moody group $\widehat{M G}$. This theorem gives a complete description of the Adjoint action iff $r_{d} \neq 0$. 
Surprisingly in the remaining case $r_{d}=0$ the situation is different: now the Adjoint action is reduced to the equations:

$$
\begin{aligned}
& \operatorname{Ad}(x) u:=g w(u) g^{-1}+\left\langle g w(u) g^{-1}, g^{\prime} g^{-1}\right\rangle c, \\
& \operatorname{Ad}(x) c:=c .
\end{aligned}
$$

Calculate the orbit of the constant function $u \equiv 0 . u$ is fixed by the Adjoint action as $\operatorname{Ad}(x) u:=g 0 g^{-1}+\left\langle g 0 g^{-1}, g^{\prime} g^{-1}\right\rangle c=0$. Hence iff we can describe the restriction of the Adjoint action to $X \mathfrak{g}^{\sigma}$ as some kind of polar action then the associated Weyl group has to be necessarily of spherical type. Furthermore the action is clearly not proper Fredholm. Hence the Hilbert-space version is not covered by Terng's results.

We use the regularity independent notation:

We define a flat of finite type to be a flat $\mathfrak{t} \subset L(\mathfrak{g}, \sigma)$ such that $\mathfrak{t}$ is the restriction of a flat in $\widehat{L}(\mathfrak{g}, \sigma)$. Hence all flats of finite type are conjugate in $\widehat{L}(G, \sigma)$ and as the orbits of $\widehat{L}(G, \sigma)$ and $L(G, \sigma)$ coincide on $L(\mathfrak{g}, \sigma)$ also in $L(G, \sigma)$. Hence any flat of finite type in $L(\mathfrak{g}, \sigma)$ is isomorphic to $\mathfrak{t}_{0} \subset \mathfrak{g}$, where we choose $\mathfrak{g}$ to denote the subalgebra of constant loops. Using the usual notion for regular and singular elements we find that the associated Weyl group is the spherical Weyl group of $\mathfrak{g}$.

REMARK 7.11. From a geometric point of view this different behavior is related to the fact that the hyperplane defined by $r_{d}=0$ corresponds to the spherical building at infinity while the space $r_{d} \neq 0$ corresponds to the spherical building at infinity. For further details confer [Fre09], [Fre12a], [Fre12b].

\section{REFERENCES}

[BBI01] D. Burago, Y. Burago, and S. Ivanov, A Course in Metric Geometry, volume 33 of Graduate Studies in Mathematics, AMS, Providence, 2001.

[BCO03] J. Berndt, S. Console, And C. Olmos, Submanifolds and holonomy, volume 434 of Research Notes in Mathematics, Chapman \& Hall, Boca Raton, 2003.

[Ber03] M. Berger, A panoramic view of Riemannian Geometry, Springer Verlag, Berlin, 2003.

[Ber08] W. Bertram, Differential geometry, Lie groups and symmetric spaces over general base fields and rings, Mem. Amer. Math. Soc., 192 (2008), $\mathrm{x}+202$.

[BG91] C. A. Berenstein and R. Gay, Complex Variables - An Introduction, volume 125 of Graduate Texts in Mathematics, Springer Verlag, New York, 1991.

[BGN04] W. Bertram, H. Glöckner, And K.-H. Neeb, Differential calculus over general base fields and rings, Expo. Math., 22 (2004), pp. 213-282.

[BM87] P. Breitenlohner and D. Maison, On the Geroch group, Ann. Inst. H. Poincaré Phys. Théor., 46 (1987), pp. 215-246.

[Car02] R. CARTER, Lie Algebras of Finite and Affine Type, volume 96 of Cambridge studies in advances mathematics, Cambrigde university press, Cambridge, 2002.

[Chu12] C.-H. CHU, Jordan structures in geometry and analysis, volume 190 of Cambridge Tracts in Mathematics, Cambridge University Press, Cambridge, 2012.

[Dad85] J. DADOK, Polar coordinates induced by actions of compact lie groups, Trans. Amer. Math. Soc., 288 (1985), pp. 125-137.

[DK00] J. J. Duistermant and J. A. C. Kolk, Lie groups, Universitext. Springer-Verlag, Berlin, 2000.

[Fre09] W. FREYN, Kac-Moody symmetric spaces and universal twin buildings, PhD thesis, Universität Augsburg, 2009.

[Fre12a] W. FreYn, Kac-Moody geometry, In "Global Differential Geometry", pp. 55-92. Springer, Heidelberg, 2012.

[Fre12b] W. FREYN, Kac-Moody groups, infinite dimensional differential geometry and cities, Asian Journal of Mathematics, 16 (2012), pp. 607-636.

[Fre12c] W. Freyn, Tame Fréchet submanifolds of Co-Banach type, accepted for publication in "Forum Mathematicum". 
[Fre13a] W. Freyn, Geometry of Kac-Moody symmetric spaces, submitted, 2013.

[Fre13b] W. FREYN, Inverse limit constructions for affine Kac-Moody groups, in preparation, 2013.

[Fre13c] W. Freyn, Orthogonal-Symmetric affine Kac-Moody-algebras, to be published in Transactions of the AMS, 2013.

[Fre13d] W. FREYN, Spherical buildings for twin cities, in preparation, 2013.

[Glö07] H. GLÖCKNER, Implicit functions from topological vector spaces to Fréchet spaces in the presence of metric estimates, arXiv:math.GM/0612673 v5, 2007.

[Gro97] A. Grothendieck, Esquisse d'un programme, In "Geometric Galois actions, 1", volume 242 of London Math. Soc. Lecture Note Ser., pp. 5-48. Cambridge Univ. Press, Cambridge, 1997. With an English translation on pp. 243-283.

[Gro00] C. Gross, s-Representations for involutions on affine Kac-Moody algebras are polar, Manuscripta Math., 103 (2000), pp. 339-350.

[Ham82] R. S. Hamilton, The inverse function theorem of Nash and Moser, Bull. Amer. Math. Soc. (N.S.), 7 (1982), pp. 65-222.

[Hei08] E. Heintze, Real forms and finite order automorphisms of affine KacMoody algebras - an outline of a new approach, http://opus.bibliothek.uniaugsburg.de/opus4/frontdoor/index/index/docId/641, 2008.

[HG09] E. Heintze AND C. Gross, Involutions, finite order automorphisms and real forms of affine Kac-Moody algebras, 2009.

[HG12] E. HeIntze AND C. GRoss, Finite order automorphisms and real forms of affine KacMoody algebras in the smooth and algebraic category, Mem. Amer. Math. Soc., 219 (2012), viii+66.

[HL99] E. HeINTZE AND X. LIU, Homogeneity of infinite-dimensional isoparametric submanifolds, Ann. of Math. (2), 149 (1999), pp. 149-181.

[HPTT95] E. Heintze, R. S. Palais, C.-L. Terng, and G. Thorbergsson, Hyperpolar actions on symmetric spaces, pp. 214-245. Conf. Proc. Lecture Notes Geom. Topology, IV. Int. Press, Cambridge, MA, 1995.

[Kö0] K. Königsberger, Analysis 2, Springer Verlag, Berlin, 2000.

[Kac68] V. G. KAC, Simple Irreducible Graded Lie Algebras of Finite Growth (russian), Math. USSR-Izvestiya, 2 (1968), pp. 1271-1311.

[Kac90] V. G. KAC, Infinite-dimensional Lie algebras, Cambridge University Press, Cambridge, third edition, 1990.

[Kan70] I. L. Kantor, Graded Lie algebras (russian), Trudy Sem. Vektor. Tenzor. Anal., 15 (1970), pp. 227-266.

[KM97] A. KRIEGL AND P. W. Michor, The convenient setting of global analysis, volume 53 of Mathematical Surveys and Monographs, American Mathematical Society, Providence, RI, 1997.

[Kna96] A. KNAPP, Lie Groups Beyond an Introduction, volume 140 of Progress in Mathematics, Birkhäuser Verlag, Boston, 1996.

[Kob11] S. Kobayashi, Real forms of complex surfaces of constant mean curvature, Trans. Amer. Math. Soc., 363 (2011), pp. 1765-1788.

[KP85] V. G. KAC AND D. H. PETERSON, Defining relations of certain infinite-dimensional groups, Astérisque, (Numero Hors Serie), pp. 165-208, 1985. The mathematical heritage of Élie Cartan (Lyon, 1984).

[KW09] B. KHESIN AND R. WENDT, The geometry of infinite-dimensional groups, volume 51 of "Ergebnisse der Mathematik und ihrer Grenzgebiete. 3. Folge. A Series of Modern Surveys in Mathematics [Results in Mathematics and Related Areas. 3rd Series. A Series of Modern Surveys in Mathematics]", Springer-Verlag, Berlin, 2009.

[Lan99a] S. LANG, Complex Analysis, volume 103 of Graduate Texts in Mathematics, Springer Verlag, New York, 1999.

[Lan99b] S. LANG, Fundamentals of differential geometry, volume 191 of Graduate Texts in Mathematics, Springer-Verlag, New York, 1999.

[Moo69] R. V. Moody, Euclidean Lie algebras, Canad. Jour. Math., 21 (1969), pp. 1432-1454.

[MP95] R. V. Moody And A. PiAnzola, Lie algebras with triangular decomposition, John Wiley Sons, New York, 1995.

[Mül06] O. MülleR, Bounded Fréchet geometry, arXiv.org:math/0612379, 2006.

[Mül08] O. Müller, A metric approach to Fréchet geometry, J. Geom. Phys., 58 (2008), pp. $1477-1500$.

[Nee06] K.-H. NeEB, Towards a Lie theory of locally convex groups, Jpn. J. Math., 1 (2006), pp. 291-468.

[NY02] D. NOVIKOV AND S. YAKOVEnKo, Lectures on meromorphic flat connections, 
math.CA/0212334, page 46, 2002.

[Omo97] H. OMORI, Infinite dimensional Lie groups, American Matheamtical Society, Providence, 1997. Translations of Mathematical Monographs, Vol. 158.

[Pal68] R. S. Palais, Foundations of Global non-linear Analysis, W. A. Benjamin, Inc., New York, 1968.

[Pop05] B. Popescu, Infinite dimensional symmetric spaces, thesis, University of Augsburg, Augsburg, 2005.

[Pop06] B. Popescu, On the geometry of infinite dimensional Lie groups, preprint, 2006.

[PS86] A. Pressley and G. Segal, Loop groups, Oxford Mathematical Monographs. The Clarendon Press Oxford University Press, New York, 1986. Oxford Science Publications.

[PT88] R. PAlais And C.-L. Terng, Critical Point Theory and Submanifold Geometry, volume 1353 of "Lecture Notes in Mathematics", Springer Verlag, Berlin, 1988.

[PT90] U. Pinkall and G. Thorbergsson, Examples of infinite-dimensional isoparametric submanifolds, Math. Z., 205 (1990), pp. 279-286.

[Rou89a] G. Rousseau, Almost split K-forms of Kac-Moody algebras, in "Infinite-dimensional Lie algebras and groups (Luminy-Marseille, 1988)", volume 7 of Adv. Ser. Math. Phys., pp. 70-85. World Sci. Publ., Teaneck, NJ, 1989.

[Rou89b] G. Rousseau, Formes réelles presque-compactes des algèbres de Kac-Moody affines, in "Algèbres de Kac-Moody affines", volume 11 of Inst. Élie Cartan, pp. 175-205. Univ. Nancy, Nancy, 1989.

[Str06] M. STROPPEL, Locally compact groups, EMS Textbooks in Mathematics. European Mathematical Society (EMS), Zürich, 2006.

[SW85] G. Segal AND G. Wilson, Loop groups and equations of KdV type, Publications Mathématiques de l'IHÉS, 61 (1985), pp. 5-65.

[Ter89] C.-L. Terng, Proper Fredholm submanifolds of Hilbert space, J. Differential Geom., 29 (1989), pp. 9-47.

[Ter95] C.-L. Terng, Polar actions on Hilbert space, J. of Geom. Anal., 5 (1995), pp. 129-150.

[Tit87] J. Tits, Uniqueness and presentation of Kac-Moody groups over fields, Journal of Algebra, 105 (1987), pp. 542-573.

[Tit89] J. Tits, Groupes associés aux algèbres de Kac-Moody, Astérisque, (177-178):Exp. No. 700, pp. 7-31, 1989. Séminaire Bourbaki, Vol. 1988/89.

[Var84] M. Varadarajan, Lie Groups, Lie Algebras, and their Representation, volume 102 of "Graduate Texts in Mathematics", Springer Verlag, New York, 1984. 